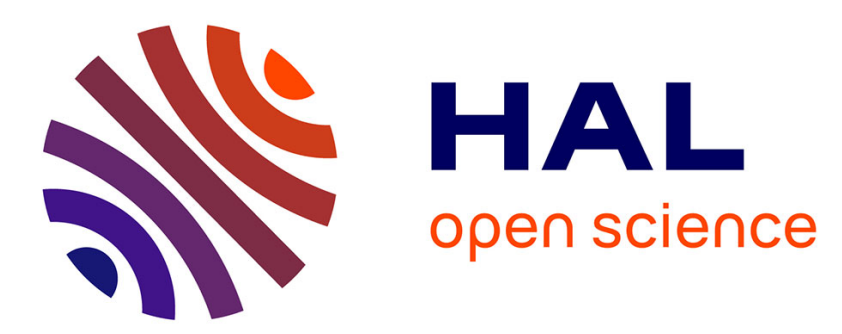

\title{
Impact of attenuation on guided mode wavenumber measurement in axial transmission on bone mimicking plates
}

Jean-Gabriel Minonzio, Josquin Foiret, Maryline Talmant, Pascal Laugier

\section{- To cite this version:}

Jean-Gabriel Minonzio, Josquin Foiret, Maryline Talmant, Pascal Laugier. Impact of attenuation on guided mode wavenumber measurement in axial transmission on bone mimicking plates. Journal of the Acoustical Society of America, 2011, 130 (6), pp.3574 - 3574. 10.1121/1.3652884 . hal-01394307

\section{HAL Id: hal-01394307 https://hal.sorbonne-universite.fr/hal-01394307}

Submitted on 9 Nov 2016

HAL is a multi-disciplinary open access archive for the deposit and dissemination of scientific research documents, whether they are published or not. The documents may come from teaching and research institutions in France or abroad, or from public or private research centers.
L'archive ouverte pluridisciplinaire HAL, est destinée au dépôt et à la diffusion de documents scientifiques de niveau recherche, publiés ou non, émanant des établissements d'enseignement et de recherche français ou étrangers, des laboratoires publics ou privés. 


\section{Impact of attenuation on guided mode wavenumber}

\section{measurement in axial transmission on bone mimicking}

plates

Jean-Gabriel Minonzio, ${ }^{\text {a) }}$ Josquin Foiret, Maryline Talmant, and Pascal Laugier

${ }^{1}$ CNRS, UPMC Univ Paris 06, UMR 7623, LIP, 15 rue de l’école de médecine F-75006, Paris, France

running title: anisotropic and absorbing wave guide

a) Author to whom correspondence should be addressed. Electronic mail:

jean-gabriel.minonzio@upmc.fr

June $11^{\text {th }} 2011$

Revision: September $15^{\text {th }} 2011$ 


\section{Abstract}

Robust signal processing methods adapted to clinical measurements of guided modes are required to assess bone properties such as cortical thickness and porosity. Recently, an approach based on the singular value decomposition (SVD) of multidimensional signals recorded with an axial transmission array of emitters and receivers has been proposed for materials with negligible absorption [Minonzio et al. J. Acoust. Soc. Am. 127, 2913-2919 (2010)]. In presence of absorption, the ability to extract guided mode degrades. The objective of the present study is to extend the method to the case of absorbing media, considering attenuated plane waves (complex wavenumber). The guided mode wavenumber extraction is enhanced and the order of magnitude of the attenuation of the guided mode is estimated. Experiments have been carried out on $2 \mathrm{~mm}$ thick plates in the $0.2-2 \mathrm{MHz}$ bandwidth. Two material are inspected: PMMA (isotropic with absorption) and artificial composite bones (Sawbones, Pacific Research Laboratory Inc, Vashon WA) which is a transverse isotropic absorbing medium. Bulk wave velocities and bulk attenuation have been evaluated from transmission measurements. These values were used to compute theoretical Lamb mode wavenumbers which are consistent with the experimental ones obtained with the SVD-based approach.

PACS numbers

4320Ye Measurement methods and instrumentation, 4380Vj Acoustical medical instrumentation and measurement techniques 4320Mv Waveguides, wave propagation in tubes and ducts 4360Fg Acoustic array systems and processing, beam-forming 


\section{INTRODUCTION}

Since more than ten years, guided waves have been known to be supported at ultrasonic frequencies by long cortical bones, such as tibia or radius., 2 The frequencydependent phase/group guided mode velocities, which are determined by bone elastic or geometrical properties, have thus the potential to be used as surrogate markers of bone strength. To date, in most in vivo axial transmission measurements, attention was focused on the measurement of the first arrival signal, denoted FAS. It has been measured on the leg (tibia) $)^{3-6}$ or the forearm (radius). ${ }^{6-9}$ Yet, measurements of dispersion curves have not been thoroughly investigated in vivo, except in a pioneering study on tibia dedicated to $\mathrm{A}_{0}$ mode. ${ }^{6}$

${ }^{10}$ However, $\mathrm{A}_{0}$ mode is more susceptible than other Lamb waves to coupling with soft tissue. $^{11}$

In vitro measurements of frequency dependent guided modes were achieved on bone specimens. Several authors used a fixed transmitter and a mobile receiver on bovine tibia. Different frequency excitation and signal processing techniques have been considered. Lefebvre et al. ${ }^{12}$ used the spatio-temporal Fourier transform at $0.1 \mathrm{MHz}$ and identified three plate or Lamb modes: $\mathrm{A}_{0}, \mathrm{~S}_{0}$ and $\mathrm{A}_{1}$. Ta et al. ${ }^{13}$ used the phase spectrum method with ultrasonic frequencies of 0.5 and $1 \mathrm{MHz}$. Three longitudinal tube modes were measured: $L(0,1), L(0,2)$ and $L(0,3)$. Le et al. ${ }^{14}$ applied seismological principles at $1 \mathrm{MHz}$ and identified different propagation paths, combination of the $\mathrm{P}$ and $\mathrm{S}$ waves. However, in all these approaches, the signals transmitted through the cortical layer along the bone axis must be recorded over a sufficiently large propagation distance (defined by the receiver scanning distance in case of a single receiver or by the length of the receiver array) in order to allow a clear separation of the different signals components in the time domain. Likewise, a large propagation distance is necessary to allow a clear separation in the frequency domain. Typical propagation distances reported in in vitro experimental works are between 60 and $160 \mathrm{~mm}$, 
which precludes the direct application of these methods to clinical measurements. The bone segment that can reasonably be investigated is limited to approximately 20 to $30 \mathrm{~mm}$ due to strong bone heterogeneities and variations of soft tissue thickness along the receiving area. Time-frequency analysis with a single transmitter - receiver configuration, has been proposed to overcome the need for an extended propagation distance. ${ }^{15,16}$ When applied on a sheep tibia, Protopappas et al. ${ }^{15}$ were able to identify several plate or Lamb guided modes $\mathrm{A}_{0}, \mathrm{~S}_{0}$, $\mathrm{A}_{1}, \mathrm{~S}_{1}$ and $\mathrm{A}_{2}$. Accurate model and advanced extraction tools ${ }^{17}$ are required to identify individual guided modes.

To overcome these limitations, a signal processing method has recently been proposed taking advantage of a multi-emitter and multi-receiver axial transmission configuration. ${ }^{18,} 19$ At each frequency $f$ of the bandwidth, the most energetic singular vectors of the response matrix are interpreted as the signal subspace basis. The so-called Norm-function corresponds to the projection into the signal subspace of non attenuated plane waves (real wavenumber $k$ ). Guided modes are associated with Norm maxima in the $(f-k)$ domain. The method has been validated for non dissipative materials such as copper plate, ${ }^{18,}{ }^{19}$ using probes initially designed for FAS clinical measurements. ${ }^{20}$ In this case, measured Norm maxima values are closed to 1.

In presence of absorption, the magnitude of the maxima of the Norm-function decreases, which degrades the ability to extract guided modes. The objective of the present study is to extend the method to the case of absorbing media such as cortical bone. The Normfunction is generalized by considering attenuated plane waves (complex wavenumber $k+\mathrm{i} \alpha$ ). Consequently, maxima are extracted in a three dimensional $(f-k-\alpha)$ domain. The Norm maxima are shown to be enhanced, allowing more reliable measurements of dispersion curves and assessment of the order of magnitude of the attenuation of the guided modes. This method is presented in Sec. II. Material and methods are then described in Sec. III. Finally 
experimental validation is presented in Sec. IV. Experiments are performed on polymethylacrylate (PMMA) and bone mimicking plates. Experimental complex guided mode wavenumbers are compared with theoretical predictions. Finally, for one particular case the results compared with the generalized Norm-function are compared and discussed with those obtained with the former Norm-function formalism and with the spatio-temporal Fourier transform.

\section{MEASUREMENT CONFIGURATION AND SIGNAL PROCESSING}

\section{A. Axial transmission configuration}

The clinical probe contains $N^{\mathrm{E}}$ emitters and $N^{\mathrm{R}}$ receivers, placed in the axial transmission configuration: emitters and receivers are aligned along the $\left(\mathrm{Ox}_{3}\right)$ axis and in contact with the wave guide to be characterized (Fig. 1). The waveguide thickness, the reception array length and the distance between the first emitters and the first receiver, are respectively denoted $e, L$ and $D$. The emitter and receiver positions are denoted $x_{j}^{\mathrm{E}}$ and $x_{j}^{\mathrm{R}}$ and satisfy $x_{i}^{\mathrm{E}}=i p$ and $x_{j}^{\mathrm{R}}=D+j p$ respectively, with $p$ the array pitch. Normal stress excitation is assumed. The length of the transducer elements along the $\left(\mathrm{Ox}_{2}\right)$ direction is large compared to the transducer elements width along the $\left(\mathrm{Ox}_{3}\right)$ direction, i.e., the array pitch. It implies that the problem can be simplified and considered as being two dimensional. An inter-element temporal response, with $j$ and $i$ the receiver and emitter indexes, is denoted $r_{j i}(t)$. Its temporal Fourier transform, denoted $R_{j i}(f)$, corresponds to an element of the $N^{\mathrm{R}} \times N^{\mathrm{E}}$ response matrix $\mathbf{R}(f)$. 


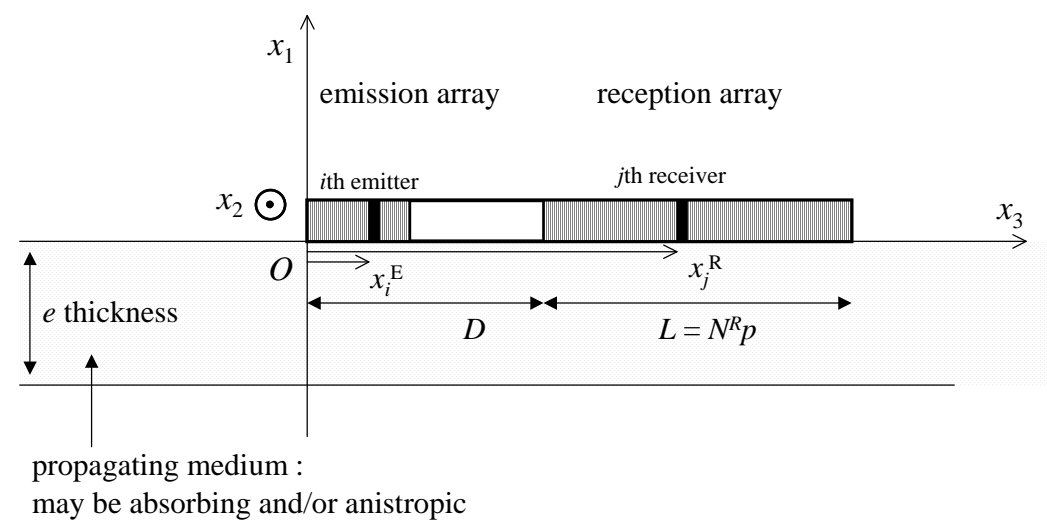

FIG. 1. Geometry of the problem: emission and reception arrays are placed in the axial transmission configuration.

\section{B. Response matrix}

An element $R_{j i}(f)$ of the theoretical response matrix $\mathbf{R}(f)$, can be written as a sum of normal modes ${ }^{21}$

$$
R_{i j}(f)=\sum_{n=1}^{N^{\mathrm{mode}}} w_{n} e^{-\alpha_{n}(f)\left(x_{j}^{\mathrm{R}}-x_{i}^{\mathrm{E}}\right)} e^{\mathrm{i} k_{n}(f)\left(x_{j}^{\mathrm{R}}-x_{i}^{\mathrm{E}}\right)},
$$

with $k_{n}(f)$ and $\alpha_{n}(f)$ the real and imaginary parts of the guided mode wavenumber. The number of guided modes is denoted $N^{\text {mode }}$ at the frequency $f$. The modal amplitude is denoted $w_{n}$ and can be given, for instance, for a non absorbing plate, assuming that normal displacement is measured at the receivers by ${ }^{21}$

$$
w_{n}=B(f)\left(\frac{p}{2} \operatorname{sinc}\left[k_{n} \frac{p}{2}\right]\right)^{2} E_{n}\left(k_{n}, f, c_{\mathrm{L}}, c_{\mathrm{T}}, e\right) \text {. }
$$

The term $B(f)$ corresponds to the frequency bandwidth. The sinc function corresponds to the transducer aperture function. The term $E_{n}$ is the intrinsic excitability of the $n^{\text {th }}$ mode. It can be calculated either using integral transform or reciprocity relationship. $^{21}$ 
Due to the two dimensional assumption, no geometric dispersion is considered as the propagation is purely in $\left(\mathrm{Ox}_{1} x_{3}\right)$ plane. Thus the amplitude decay is due to the guided mode attenuation and varies as $e^{-\alpha_{n}(f) x_{3}}$. Transducers of finite dimension along the $\left(O x_{2}\right)$ axis have been described in Ref. ${ }^{22}$ and are not considered here.

Considering the two previous equations, the theoretical response matrix can be written $\mathbf{R}(f)=\mathbf{a}^{\mathrm{R}} \mathbf{W}^{t} \mathbf{a}^{\mathrm{E}^{*}}$. The notation ${ }^{t}$ and ${ }^{*}$ correspond to the transpose and complex conjugation operations. Elements of the reception and emission matrices $\mathbf{a}^{\mathrm{R}}$ and $\mathbf{a}^{\mathrm{E}}$ are

$$
\begin{aligned}
& a_{j n}^{\mathrm{R}}=\exp \left(\left[-\alpha_{n}(f)+\mathrm{i} k_{n}(f)\right] x_{j}^{\mathrm{R}}\right), \\
& a_{i n}^{\mathrm{E}}=\exp \left(\left[\alpha_{n}(f)+\mathrm{i} k_{n}(f)\right] x_{i}^{\mathrm{E}}\right) .
\end{aligned}
$$

The matrix $\mathbf{W}$ is diagonal and contains the weighting terms $w_{n}$ [Eq.(2)], i.e., $W_{n m}=w_{n} \delta_{n m}$ with $\delta_{n m}$ the Kronecker symbol. The theoretical response matrix can be expressed using normalized vectors as

$$
\mathbf{R}(f)=\mathbf{e}^{\mathrm{R}} \mathbf{S}^{t} \mathbf{e}^{\mathrm{E}^{*}}
$$

The normalized guided mode vectors in emission or reception satisfy

$$
\mathbf{e}_{n}^{\mathrm{E}, \mathrm{R}}=\frac{\mathbf{a}_{n}^{\mathrm{E}, \mathrm{R}}}{\left\|\mathbf{a}_{n}^{\mathrm{E}, \mathrm{R}}\right\|} .
$$

The notation $\left\|\mathbf{a}^{\mathrm{E}}\right\|$ corresponds to the norm of the $\mathbf{a}_{n}^{\mathrm{E}}$ vector given by Eq. (3). Its expression is given in Eq. (A3) of the appendix. With $N^{\text {mode }}$ the number of guided modes at the frequency $f$, the dimensions of the reception and emission normalized guided mode matrices $\mathbf{e}^{\mathrm{R}}$ and $\mathbf{e}^{\mathrm{E}}$ are $N^{\mathrm{R}} \times N^{\text {mode }}$ and $N^{\mathrm{E}} \times N^{\text {mode }}$ respectively. The $N^{\text {mode }} \times N^{\text {mode }}$ matrix $\mathbf{S}$ is diagonal and contains the normalized weighting terms

$$
S_{n}=w_{n}\left\|\mathbf{a}_{n}^{\mathrm{R}}\right\|\left\|\mathbf{a}_{n}^{\mathrm{E}}\right\| .
$$




\section{Norm-function expression}

In a first step, the singular value decomposition (SVD) is applied to the response matrix $\mathbf{R}(f)$. The SVD of the response matrix writes as a three matrix product

$$
\mathbf{R}(f)=\mathbf{U} \Sigma^{t} \mathbf{V}^{*}
$$

The matrices $\mathbf{U}$ and $\mathbf{V}$ contain the reception and emission singular vectors. The real positive diagonal matrix $\Sigma$ contains the singular values. The SVD outputs used in the following are the singular values $\sigma_{m}(f)$ and the reception singular vectors $\mathbf{U}_{m}(f)$. The number of experimental singular values and vectors is equal to the minimum of $N^{\mathrm{E}}$ and $N^{\mathrm{R}}$, i.e., $N^{\mathrm{E}}$ in this study.

The most energetic singular values are used to define the signal subspace, whereas the other singular values define the complementary noise subspace. To this end, a first adaptive threshold $t_{1}$ is used. Only singular values higher than the threshold $t_{1}$ are kept and associated with the signal subspace. The threshold value is heuristically chosen at each frequency. The number of kept singular values corresponds to the rank of the signal subspace and is denoted $M$. The rank of the signal subspace is less or equal than $N^{\text {mode }}$ at each frequency. Thus the signal singular vectors subspace $\operatorname{Span}\left(\mathbf{U}_{m \leq M}\right)$ is included in the guided mode subspace

$\operatorname{Span}\left(\mathbf{e}_{n}{ }^{\mathrm{R}}\right)$. The singular vectors associated with the signal subspace, i.e., $\mathbf{U}_{m \leq M}$, form the received signal basis.

Consider an attenuated spatial plane wave with a complex wavenumber $k+i \alpha$, denoted $\mathbf{e}^{\text {test }}(k, \alpha)$. It is defined as the test normalized guided mode following Eqs. (3) and (5) as

$$
\mathrm{e}^{\mathrm{test}}(k, \alpha)=\mathrm{e}^{\mathrm{R}}(k, \alpha) .
$$

The test vector $\mathbf{e}^{\text {test }}(k, \alpha)$ is expressed in the canonical basis , i.e., the receiver basis [Eq. (3)]. It can be projected in the signal subspace, using the singular vector basis, as 


$$
\left.\mathbf{e}^{\text {test }}\right|_{\text {signal }}=\sum_{m=1}^{M}\left\langle\mathbf{U}_{m} \mid \mathbf{e}^{\text {test }}\right\rangle \mathbf{U}_{m} .
$$

The Hermitian scalar product $<\mathbf{U}_{m} \mid \mathbf{e}^{\text {test }}>$ is equal to ${ }^{t} \mathbf{U}_{m}{ }^{*}$. ${ }^{\text {test }}$. The Norm-function is defined at each frequency, as the square of the norm of the previous expression

$$
\operatorname{Norm}(f, k, \alpha)=\sum_{m=1}^{M}\left|\left\langle\mathbf{U}_{m}(f) \mid \mathbf{e}^{\text {test }}(k, \alpha)\right\rangle\right|^{2} \text {. }
$$

The Norm-function is real positive. Due to the normalization [Eqs. (5) and (7)], it ranges from 0 to 1 . Equation (10) corresponds to the extension of the non attenuated case. ${ }^{18}$ In this previous formalism, the Norm-function was expressed in the two dimensional $(f, k)$ plane. Here, the Norm-function is expressed in the three dimensional $(f, k, \alpha)$ space, where $\alpha$ is the imaginary part of the testing wavenumber. Thus, the previous definition is included in the new formalism and corresponds to the $\alpha=0$ line of a $(k, \alpha)$ plane.

\section{Norm-function interpretation}

The Norm-function can be interpreted using the guided mode basis. Equations (4) and (7) are formally similar, i.e., the response matrix $\mathbf{R}(f)$ can be expressed in function of the normalized guided modes basis $\left(\mathrm{e}_{n}{ }^{\mathrm{R}}\right)$ or in function of the signal singular vectors basis $\left(\mathrm{U}_{m \leq M}\right)$. Due to the finite reception array length, the normalized guided modes $\mathbf{e}_{n}{ }^{\mathrm{R}}$ are not orthogonal. On the contrary, the singular vectors $\mathbf{U}_{m \leq M}$ are defined orthogonal. ${ }^{23}$ Thus the singular vectors are combination of the normalized guided modes. These two basis are represented schematically in Fig. 2 in the case of a rank 2 signal subspace, i.e., $\operatorname{Span}\left(\mathbf{e}_{1}{ }^{\mathrm{R}}, \mathbf{e}_{2}{ }^{\mathrm{R}}\right)$ or $\operatorname{Span}\left(\mathbf{U}_{1}, \mathbf{U}_{2}\right)$. 


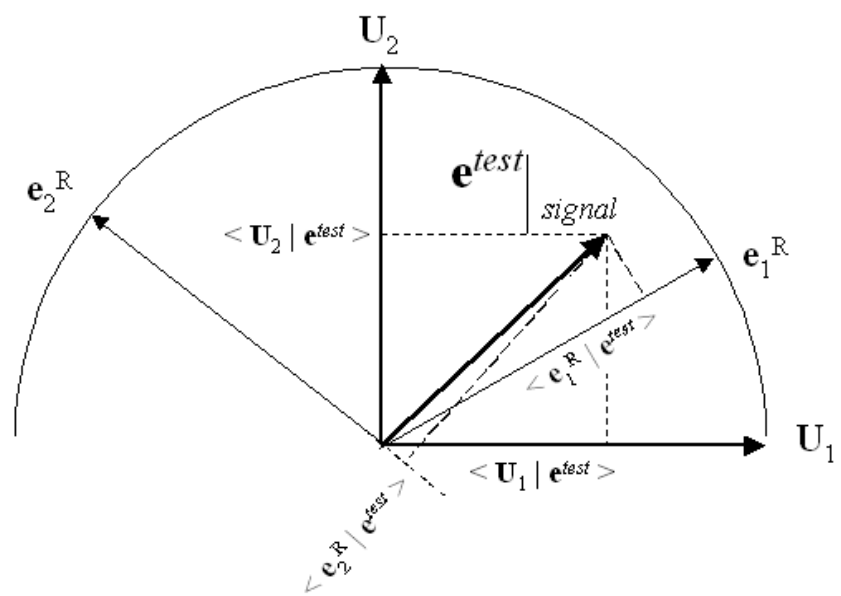

FIG. 2. Schematic representation of a signal subspace of rank 2: the test vector $\mathbf{e}^{\text {test }}$ can be projected on the singular vector basis $\left(\mathbf{U}_{1}, \mathbf{U}_{2}\right)$ [Eq. (9)] or in normalized guided mode basis $\left(\mathbf{e}_{1}{ }^{\mathrm{R}}, \mathbf{e}_{2}{ }^{\mathrm{R}}\right)$ [Eq. (11)].

The projection in the signal subspace of the test vector $\mathbf{e}^{\text {test }}$ can be expressed using the singular vector basis [Eq. (9)] or using the normalized guided mode basis as

$$
\left.\mathbf{e}^{\text {test }}(k, \alpha)\right|_{\text {signal }} \approx \sum_{n=1}^{N^{\text {mode }}}\left\langle\mathbf{e}_{n}^{\mathrm{R}} \mid \mathbf{e}^{\text {test }}\right\rangle \mathbf{e}_{n}^{\mathrm{R}} .
$$

If $k+\mathrm{i} \alpha$ is close to a guided mode wavenumber $k_{n}+\mathrm{i} \alpha_{n}$, the test vector $\mathbf{e}^{\text {test }}(k, \alpha)$ is projected mainly on the $n^{\text {th }}$ guided mode $\mathbf{e}_{n}^{\mathrm{R}}\left(k_{n}, \alpha_{n}\right)$ as

$$
\left.\mathbf{e}^{\text {test }}\left(k \sim k_{n}, \alpha \sim \alpha_{n}\right)\right|_{\text {signal }} \approx\left\langle\mathbf{e}_{n}^{\mathrm{R}}\left(k_{n}, \alpha_{n}\right) \mid \mathbf{e}^{\text {test }}\right\rangle \mathbf{e}_{n}^{\mathrm{R}}\left(k_{n}, \alpha_{n}\right),
$$

and the Norm-function [Eq. (10)] is given in that case by

$$
\operatorname{Norm}\left(f, k \sim k_{n}, \alpha \sim \alpha_{n}\right)=\left|\left\langle\mathbf{e}_{n}^{\mathrm{R}}\left(k_{n}, \alpha_{n}\right) \mid \mathbf{e}^{\text {test }}(k, \alpha)\right\rangle\right|^{2} .
$$

The expression of the Hermitian scalar product $\left\langle\mathbf{e}_{n}^{\mathrm{R}}\left(k_{n}, \alpha_{n}\right) \mid \mathbf{e}^{\text {test }}(k, \alpha)\right\rangle$ is given by Eq. (A6) in the appendix. This scalar product can be interpreted as the normalized spatial Fourier transform of the normalized guided mode $\mathbf{e}_{n}^{\mathrm{R}}\left(k_{n}, \alpha_{n}\right)$. Thus, the SVD can be interpreted as a 
denoising step included between the temporal and spatial Fourier transforms. If $k+\mathrm{i} \alpha$ corresponds to a guided mode wavenumber $k_{n}+\mathrm{i} \alpha_{n}$, the Norm-function is close to 1 . If $k+\mathrm{i} \alpha$ does not correspond to a guided mode wavenumber, the Norm-function is small compared to 1. The resolution, i.e., the ability to discriminate two close modes, corresponds to the main lobe width. $^{18}$

Thus the real and imaginary part of the guided mode wavenumbers can be obtained from the Norm maxima positions in the $(f, k, \alpha)$ 3D-space. A second threshold $t_{2}$ is used to select the retained maxima larger than $t_{2}$. As the maximum value reflects how the spatial plane wave is represented in the basis of the signal subspace, the value is interpreted as a quality parameter ranging from 0 to 1 .

As discussed in the paragraph II.C, the definition of the Norm-function adapted to the case of non-attenuating propagation media corresponds to the $\alpha=0$ 2D-restriction of the $(f, k, \alpha) 3 \mathrm{D}$-space. The 2D point of view is limited in case of absorbing waveguides, because the maxima of the $\operatorname{Norm}(f, k, 0)$ function decreases as $\alpha_{n}$ increases [Eq. (A7)]. Thus maxima can be of the order of other mode secondary maxima or simply associated with noise. Thus, in presence of absorption, the magnitude of the maxima of the 2D-Norm-function decreases, this degrades the ability to extract guided mode. On the contrary, with the 3D-Norm-function definition, the maxima are enhanced and closer to 1 . Thus a second threshold $t_{2}$ closer to 1 can be chosen. Moreover, the order of magnitude of the attenuation of the guided mode $a_{n}(f)$ is estimated. An example is shown and discussed in Sec. IV.C. 


\section{MATERIAL AND METHODS}

\section{A. Samples}

Two materials have been measured: PMMA and bone mimicking plates. The plate thicknesses, about $2 \mathrm{~mm}$, correspond to a typical cortical bone thickness. The PMMA is considered as isotropic. The bone mimicking material (Sawbones, Pacific Research Laboratory Inc, Vashon WA) is made of oriented glass fibers mixed with epoxy. The material is transverse isotropic, with elastic properties close to those of real bones. The velocities and attenuation coefficients of bulk compression and shear waves of both materials were determined with longitudinal (2.25 MHz Panametrics V105RM) and transverse (1 MHz Panametrics V152 RM) contact transducers ${ }^{24}$ using a conventional through-transmission method on 10 mm-thick plate of the same material. ${ }^{25-27}$ Constant rate of attenuation increase with frequency are experimentally observed. ${ }^{28}$ Thus, the bulk wave absorption per wavelength $(\alpha \lambda)_{\mathrm{L}, \mathrm{T}}$ is constant, where subscripts $\mathrm{L}$ and $\mathrm{T}$ refer to compression and shear waves respectively. With this notation, a bulk plane wave can be written as $\exp \left[\mathrm{i} k_{\mathrm{L}, \mathrm{T}}\left(1+\mathrm{i}(\alpha \lambda)_{\mathrm{L}, \mathrm{T}} / 2 \pi\right) x\right]$

The measured values compression $\left(c_{\mathrm{L}}\right)$ and shear $\left(c_{\mathrm{T}}\right)$ bulk velocities and compression $(\alpha \lambda)_{\mathrm{L}}$ and shear $(\alpha \lambda)_{\mathrm{T}}$ attenuation, the density $\rho$ and the plate thickness $e$ are reported in Table I. The PMMA values are consistent with previously reported values. ${ }^{29-32}$ For the bone mimicking material, the bulk velocities and attenuation are first measured perpendicular $(\perp)$ to the fibers using the $10 \mathrm{~mm}$-thick plate. In order to evaluate the longitudinal properties parallel $(\|)$ to the fibers, measurement have been done on a small cube (about $10 \times 10 \times 10.2$ $\mathrm{mm}^{3}$ ) extracted from the plate. The cube dimensions were large enough to evaluate the parallel bulk velocities $c_{\mathrm{L}}{ }^{\|}$and $c_{\mathrm{T}}{ }^{\|}$but too small to evaluate the attenuations $(\alpha \lambda)_{\mathrm{L}}{ }^{\|}$and $(\alpha \lambda)_{\mathrm{T}}{ }_{\mathrm{T}}^{\|}$. 
The measured values are consistent with previously reported values on Sawbones ${ }^{33}$ or epoxy plates. $^{34}$ These values are used to compute theoretical guided mode wavenumbers as discussed in paragraph III.B. Theoretical predictions are then compared to experimental data in Sec. IV.

TABLE I. Measured values for PMMA and bone mimicking (bone mim.) plates

\begin{tabular}{lcccccc}
\hline & $c_{\mathrm{L}}\left(\mathrm{m} . \mathrm{s}^{-1}\right)$ & $c_{\mathrm{T}}\left(\mathrm{m}^{\mathrm{s}} \mathrm{s}^{-1}\right)$ & $(\alpha \lambda)_{\mathrm{L}}(\mathrm{Np})$ & $(\alpha \lambda)_{\mathrm{T}}(\mathrm{Np})$ & $\rho\left(\mathrm{g} . \mathrm{cm}^{-3}\right)$ & $e(\mathrm{~mm})$ \\
\hline \hline PMMA & $2685 \pm 20$ & $1365 \pm 10$ & $0.059 \pm 0.007$ & $0.063 \pm 0.003$ & 1.18 & 1.95 \\
bone mim. $\perp$ & $2870 \pm 40$ & $1520 \pm 20$ & $0.087 \pm 0.013$ & $0.147 \pm 0.004$ & 1.64 & 2.30 \\
bone mim. $\|$ & $3705 \pm 40$ & $1585 \pm 20$ & & & & \\
\hline
\end{tabular}

\section{B. Lamb wave calculations}

Theoretical Rayleigh-Lamb spectrum was calculated for isotropic (PMMA) and transverse isotropic plates (bone mimicking material). ${ }^{2,}{ }^{35,}{ }^{36}$ For the bone mimicking plate, two cases are considered, depending if the probe is placed perpendicularly or parallel to the fibers. In experiments, as the probe works in piston mode and does not excite horizontal shear displacement, only motion in the plane $\left(O x_{1} x_{3}\right)$ is considered. ${ }^{37}$ Due to geometrical hypothesis discussed in paragraph II.A, the perpendicular orientation is similar to an isotropic case. Thus, in this case, an absorbing plate with linear variation of absorption with frequency was assumed. For small values of $(\alpha \lambda)_{\mathrm{L}, \mathrm{T}}$, the real part of the guided mode wavenumbers is close to the non absorbing values. If the probe is placed parallel to the fibers, transverse isotropic case is considered. Nevertheless, as the attenuation could not be measured for all stiffness coefficients (Table I), only the non absorbing transverse isotropic is considered. The $c_{13}$ coefficient was deduced from $c_{33}$ and $c_{44}$ using $c_{13}=c_{33}-2 c_{44}$. 


\section{Axial transmission measurements}

The custom made probe used in this study contains 32 receivers and 5 emitters. ${ }^{19}$ The reception array pitch, denoted $p$, is equal to $0.8 \mathrm{~mm}$. The reception array length, denoted $L$, is about $26 \mathrm{~mm}$. The probe is driven by a custom made multichannel emission and reception electronic system (Althaïs Technologie, Tours, France). Emitted signals are wideband pulses (-50 dB bandwidth $0.2-2 \mathrm{MHz}$ ). The sampling frequency is equal to $20 \mathrm{MHz}$. The number of recorded time samples is equal to 1024. Fifty times signals are averaged to increase signal-tonoise ratio.

The experimental guided mode wavenumbers are extracted from the Norm-function in the following way. In a first step, the inter-element responses $r_{j i}(t)$ are experimentally recorded. Then, the experimental response matrix $\mathbf{R}(f)$ is computed using the temporal Fourier transform for each frequency of the bandwidth. The SVD is then computed at each frequency and the heuristic threshold $t_{1}$ is applied on the singular values in order to define the signal subspace dimension $M$, as discussed in paragraph II.C. Finally, the Norm-function is computed in the $(f, k, \alpha)$ 3D-space following Eq. (10). The experimental wavenumbers are obtained from the values of the Norm-function larger than a second threshold $t_{2}$ equal here to 0.85. Experimental results are shown and discussed in Sec. IV. For one particular case, the former Norm-function, i.e., for $\alpha=0$, and the spatio-temporal Fourier transform are also performed. These results are compared with those obtained the generalized Norm-function in this particular case. 


\section{EXPERIMENTAL RESULTS}

\section{A. Guided mode wavenumbers of the PMMA plate}

First, axial transmission measurements have been carried out on the isotropic dissipative PMMA plate. The plate thickness $e$ measured with a caliper was $1.95 \mathrm{~mm}$ (Table I). The real and imaginary parts of wavenumbers $k_{n}{ }^{\exp }(f)$ and $\alpha_{n}{ }^{\exp }(f)$ are obtained from the 3D-Norm(f, $k, \alpha)$ function maxima. Results are shown on Fig. 3(a) and (b). Experimental results are compared with the theoretical Lamb guided wavenumbers computed using velocity and attenuation values shown in Table I. The modes are labeled $S_{n}$ or $A_{n}$ considering their symmetry or anti-symmetry nature and their cutoff frequency $\times$ thickness product denoted $f_{c}$.e. Five modes with transverse asymptotic behavior are identified: $\mathrm{A}_{1}\left(f_{c}\right.$.e $\left.=c_{\mathrm{T}} / 2\right), \mathrm{S}_{2}\left(c_{\mathrm{T}}\right)$, $\mathrm{A}_{3}\left(3 / 2 c_{\mathrm{T}}\right), \mathrm{S}_{4}\left(2 c_{\mathrm{T}}\right)$ and $\mathrm{A}_{5}\left(5 / 2 c_{\mathrm{T}}\right)$. Moreover, two modes with longitudinal asymptotic behavior are identified: $\mathrm{S}_{1}\left(f_{c} . e=c_{\mathrm{L}} / 2\right)$ and $\mathrm{A}_{2}\left(c_{\mathrm{L}}\right)$. The $\mathrm{S}_{0}$ mode is also measured at low frequency.

The experimental real part of the guided wavenumbers $k_{n}{ }^{\exp }(f)$ are overall in good agreement with the theoretical Lamb guided wavenumbers [Fig. 3(a)] except for $A_{3}$ around 1.4 MHz and 3.5 rad. $\mathrm{mm}^{-1}$, where the agreement is poorer. Part of the $k$ negative branch of the $\mathrm{S}_{1}$ is measured around $0.7 \mathrm{MHz}$. The mode are measured if their intrinsic excitability $E_{n}(f)$ is large enough. For example, the $\mathrm{S}_{2}$ mode is not observed between around $0.8 \mathrm{MHz}$. Similar behavior is observed for $S_{1}$ mode around 1.6 MHz. These examples are marked with a vertical arrow on Fig. 3. At these frequency, the low intrinsic excitability $E_{n}$ [Eq. (2) and Fig. 4] implies that the mode is poorly observed. Moreover, repeatability tests have been conducted. Three different measurements have been carried out at room temperature with varying pressing force exerting on the probe. The real and imaginary parts of the guided mode wavenumbers led to a coefficient of variation smaller than $1 \%$. 


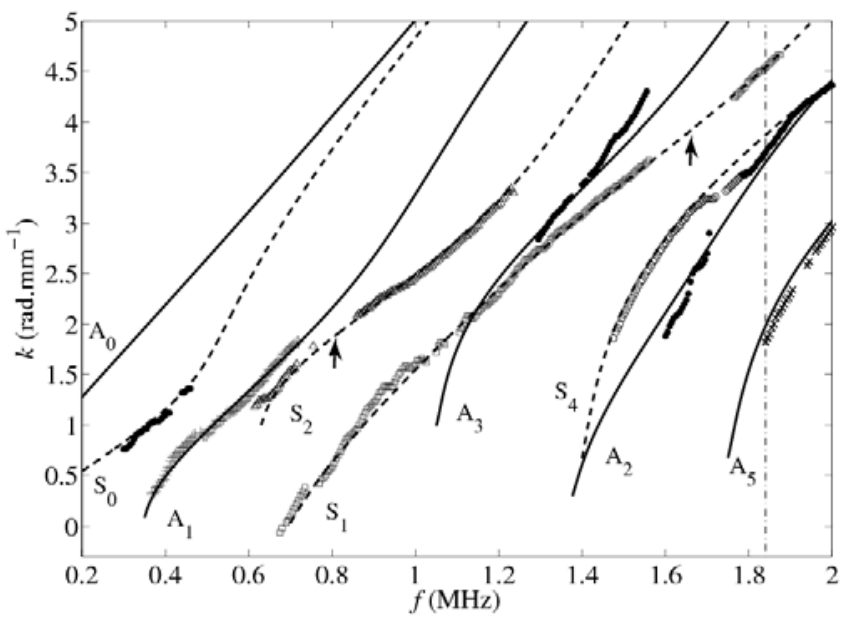

(a)

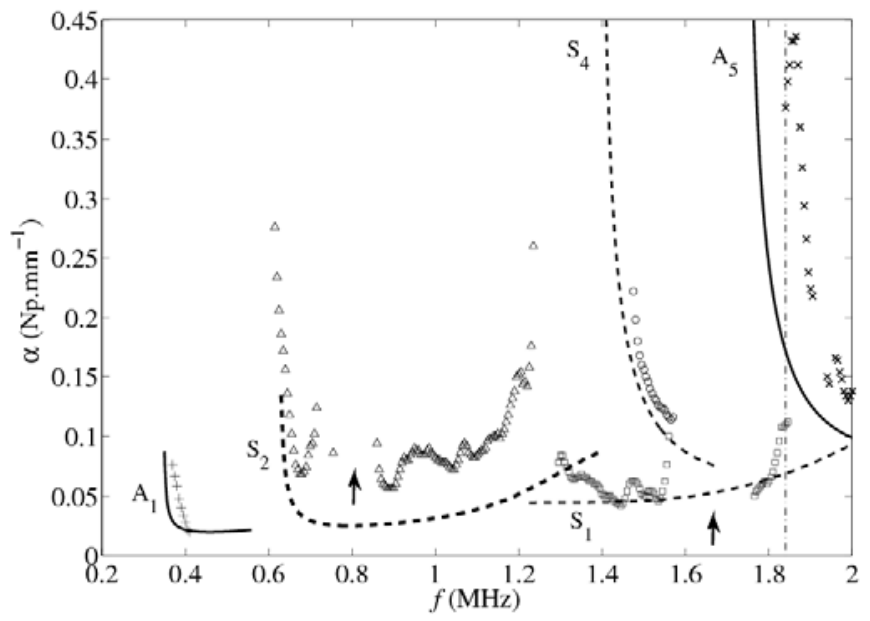

(b)

FIG. 3. Results for the PMMA plate: real (a) and imaginary parts (b) of the guided mode wavenumbers $k_{n}(f)+\mathrm{i} \alpha_{n}(f)$. Experimental values (each mode is represented with a different symbol) determined from the 3D Norm values and a threshold $t_{2}=0.85$ are compared with the theoretical Lamb modes $\mathrm{A}_{n}$ (continuous line) and $\mathrm{S}_{n}$ (dash line). For the imaginary part, four modes are shown $S_{1}, S_{2}, S_{4}$ and $A_{5}$. Arrows correspond to guided mode excitability minima. The $f=1.84 \mathrm{MHz}$ case (shown with a vertical dash-dot line) is discussed in paragraph IV.C. 


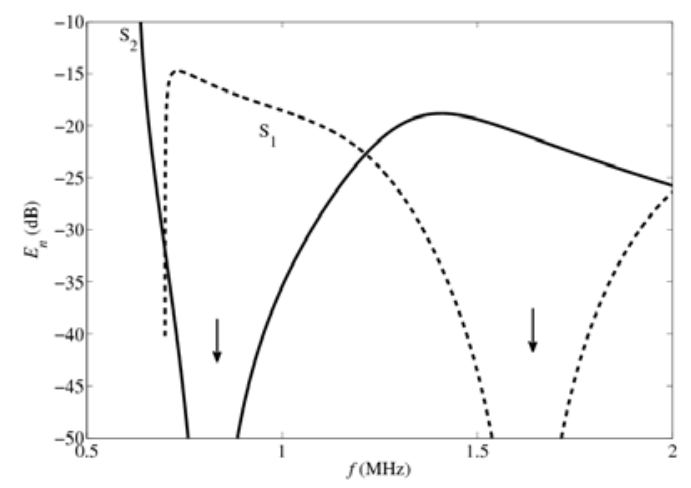

FIG. 4. Intrinsic excitability $E_{n}$ function [Eq. (2)] of a $1.95 \mathrm{~mm}$ thick PMMA plate for the $\mathrm{S}_{1}$ and $S_{2}$ modes. If the excitability is too low (as shown by the arrows), the modes are not observed.

For the experimental imaginary part of the guided wavenumbers $\alpha_{n}{ }^{\text {exp }}(f)$, the agreement with the theoretical Lamb guided wavenumbers remains reasonable, although less accurate [Fig. 3(b)] compared to the real part $k_{n}{ }^{\exp }(f)$ [Fig. 3(a)]. Nevertheless the overall behavior, as for example close to cutoff frequencies, is observed. The previous low excitability domains (i.e., $\mathrm{S}_{1}$ around $0.8 \mathrm{MHz}$ and $\mathrm{S}_{2}$ around $1.6 \mathrm{MHz}$ ) are marked with an arrow. In this domain, the attenuation is not measured. The observed deviation between experimental data and theoretical predictions may have several origins, such as attenuation sources (such as geometrical dispersion) which are not properly taken into account in the model or experimental inaccuracies in through-transmission measurements of reference attenuation values (Table I). Modes vicinity may also affect attenuation evaluation as for example $\mathrm{S}_{1}$ and $\mathrm{A}_{2}$ around $1.7 \mathrm{MHz}$. The two modes with longitudinal asymptotic behavior $\left(\mathrm{S}_{1}\right.$ and $\left.\mathrm{A}_{2}\right)$ are experimentally more attenuated than expected from the Lamb free plate spectrum. It may be due to the coupling between the probe and the wave guide which has not been taken into account in the model. 


\section{B. Guided mode wavenumbers of the bone mimicking plate}

The bone mimicking plate thickness has been next measured to $2.3 \mathrm{~mm}$ with a caliper (Table I). First, the probe is placed perpendicularly to the fibers. Due to the geometrical hypothesis described in Sections. II and III, this configuration can be considered as isotropic. Thus, an analysis similar to the PMMA case has been used. The same threshold $t_{2}=0.85$ has been used. The results are shown in Fig. 5(a) and (b). The experimental real $k_{n}{ }^{\text {exp }}(f)$ and imaginary part $\alpha_{n}^{\text {exp }}(f)$ of the guided mode wavenumber (shown with symbols) are compared with the theoretical values. The same modes are observed as in the PMMA case, but they appear in a different order, because of the value taken by the ratio $c_{\mathrm{L}} / 2 c_{\mathrm{T}}$, which is larger than 1 for PMMA and less than 1 for the bone mimicking plate measured perpendicularly to the fibers (Table I). The agreement between experimental and theoretical data is overall good and of comparable to the PMMA case, except for the mode $\mathrm{S}_{2}$ around $1.2 \mathrm{MHz}$ and for the modes $\mathrm{A}_{2}$ and $\mathrm{S}_{4}$ mode around 1.7 MHz. Observed discrepancies between experimental data and theoretical predictions may be caused by an interference between different modes, typically between $\mathrm{S}_{2}$ and $\mathrm{A}_{3}$ around $1.2 \mathrm{MHz}$ and between $\mathrm{A}_{2}$ and $\mathrm{S}_{4}$ around $1.7 \mathrm{MHz}$, respectively. 


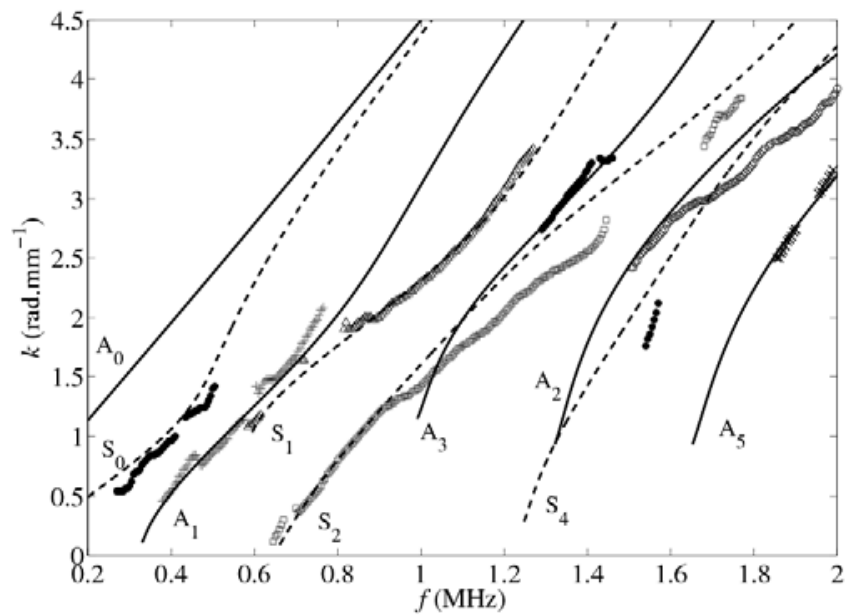

(a)

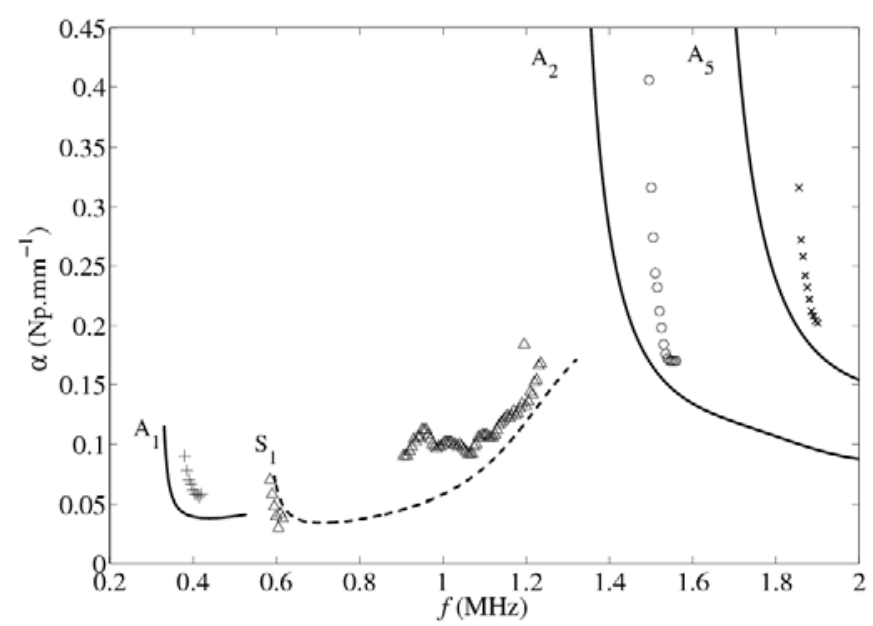

(b)

FIG. 5. Results for the $2.3 \mathrm{~mm}$ bone mimicking plate, measured with the probe oriented perpendicularly to the fibers. Real (a) and imaginary (b) parts of the guided mode wavenumbers $k_{n}(f)+\mathrm{i} \alpha_{n}(f)$. 
Finally, the probe is placed in the fiber direction, a configuration which is similar to clinical measurements. The theoretical guided mode wavenumbers are computed in the anisotropic case following paragraph III.B. The results are shown in Fig. 6. Only, the experimental real part $k_{n}{ }^{\exp }(f)$ of the guided mode wavenumber (shown with dots) are compared with the theoretical guided number wavenumbers, showing reasonable agreement experimental and theoretical data as in the previous cases. As the attenuation coefficients $(\alpha \lambda)_{\mathrm{L}}{ }^{\|}$and $(\alpha \lambda)_{\mathrm{T}}{ }_{\mathrm{T}}$ were not measured (Table I), the theoretical imaginary part of the guided mode wavenumbers was not computed.

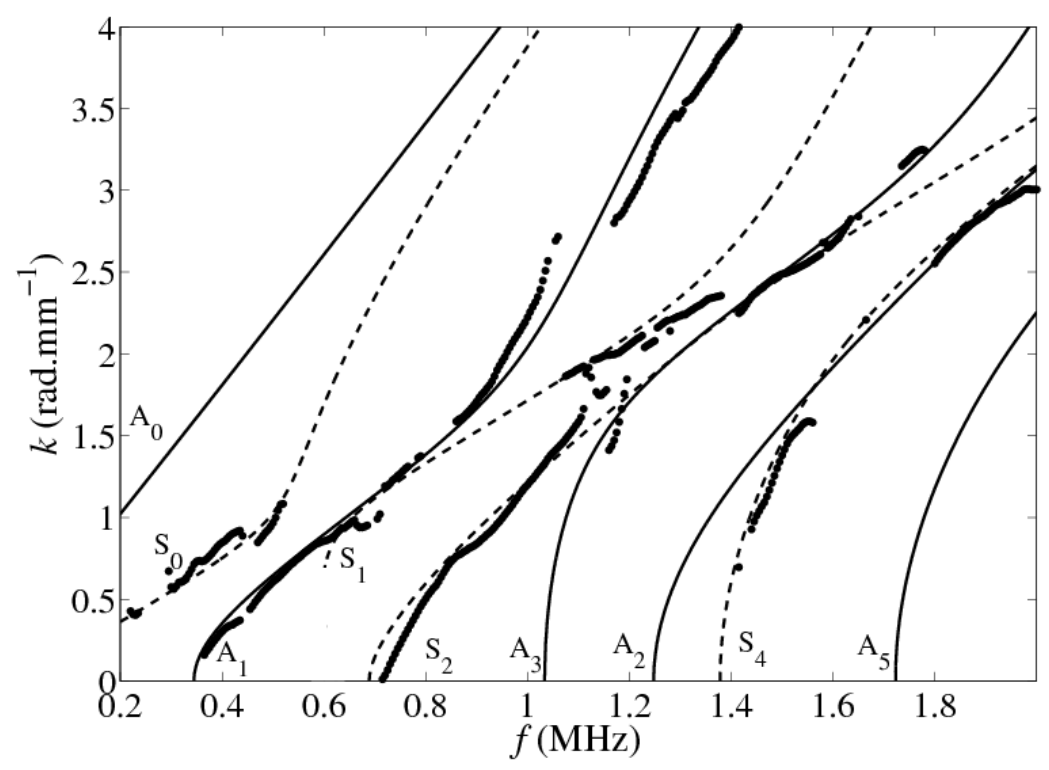

FIG. 6. Results for the $2.3 \mathrm{~mm}$ bone mimicking plate, the probe parallel to the fibers, real part $k_{n}(f)$ of the guided mode wavenumbers. 


\section{Discussion}

In order to illustrate the method, the particular case of the PMMA plate at $1.84 \mathrm{MHz}$ is now discussed. This case is marked with a vertical dash-dot line on Fig. 3. At this frequency, three guided mode are measured: $A_{5}, A_{2}$ and $S_{1}$ and the experimental rank (or signal subspace dimension) $M$ is equal to 3. The corresponding $\operatorname{Norm}(f, k, \alpha)$ function [Eq. (10)], i.e., $\left|<\mathbf{U}_{1}\right| \mathbf{e}^{\text {test }}(k, \alpha)>\left.\right|^{2}+\left|<\mathbf{U}_{2}\right| \mathbf{e}^{\text {test }}(k, \alpha)>\left.\right|^{2}+\left|<\mathbf{U}_{3}\right| \mathbf{e}^{\text {test }}(k, \alpha)>\left.\right|^{2}$, is illustrated on Fig. 7(a) in the $(k, \alpha)$ plane with $f$ being equal to $1.84 \mathrm{MHz}$. Three peaks (shown with crosses) are observed in the $(k, \alpha)$ plane corresponding to the experimental wavenumbers. The real part of the wavenumber $k_{n}^{\text {exp }}(f)$ are equal to $1.9\left(\mathrm{~A}_{5}\right), 3.7\left(\mathrm{~A}_{2}\right)$ and $4.6 \mathrm{rad} \cdot \mathrm{mm}^{-1}\left(\mathrm{~S}_{1}\right)$. The corresponding imaginary part $\alpha_{n}{ }^{\text {exp }}(f)$ equal to $0.15\left(\mathrm{~A}_{2}\right) 0.2\left(\mathrm{~S}_{1}\right)$ and $0.4 \mathrm{~Np} \cdot \mathrm{mm}^{-1}\left(\mathrm{~A}_{5}\right)$. In this example, the peak values for these three modes, range from 0.9 to 0.95 . Thus the second threshold $t_{2}$, chosen equal to 0.85 , allows to select to the peaks associated with these guided modes. This threshold is shown with a thick line on Fig. 7(a).

As attenuation increases, the useful reception length decreases. Figure 8 shows the modulus of different normalized guided modes $\mathrm{e}_{n}{ }^{\mathrm{R}}\left(k_{n}, \alpha_{n}\right)$ with $\alpha_{n}$ ranging from 0 to 0.5 rad.mm ${ }^{-1}$. For example, with $\alpha_{n}$ equal to $0.4 \mathrm{~Np} \cdot \mathrm{mm}^{-1}$, the measured amplitude at the middle of the reception array is $-40 \mathrm{~dB}$ below the amplitude of the signal measured at the first receiver. In this case, only half of the reception array can be considered as useful, the second half of received signals being with poor signal-to-noise ratio. It implies that the main lobe width increases as the attenuation increases [Eq. (A6) and Fig. 7]. The peak associated with $A_{2}$ is larger than expected due to the vicinity of the $S_{4}$ mode. The two modes are too close to be resolved. 


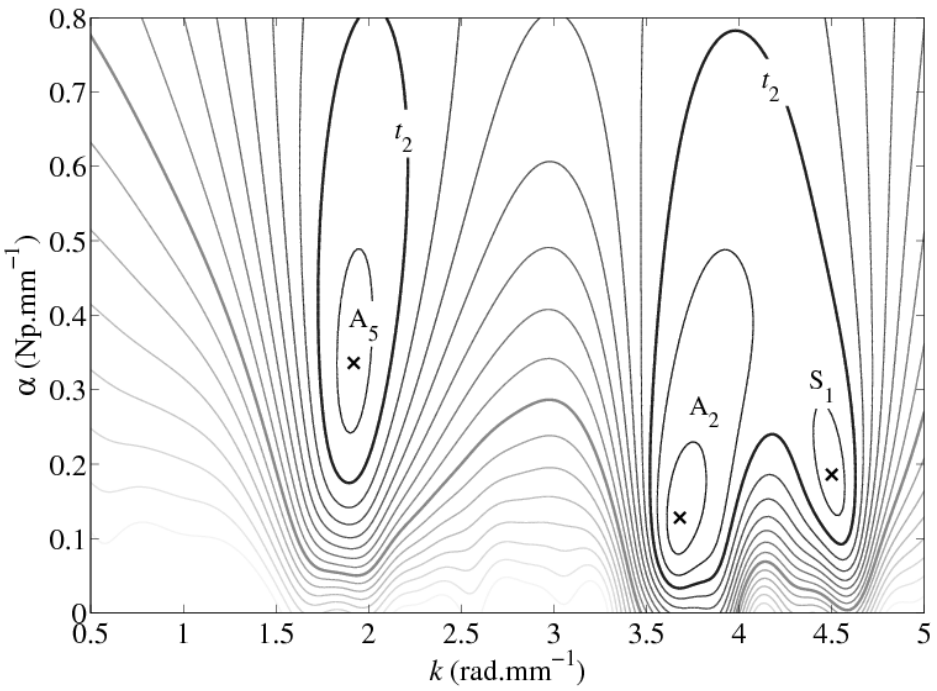

(a)

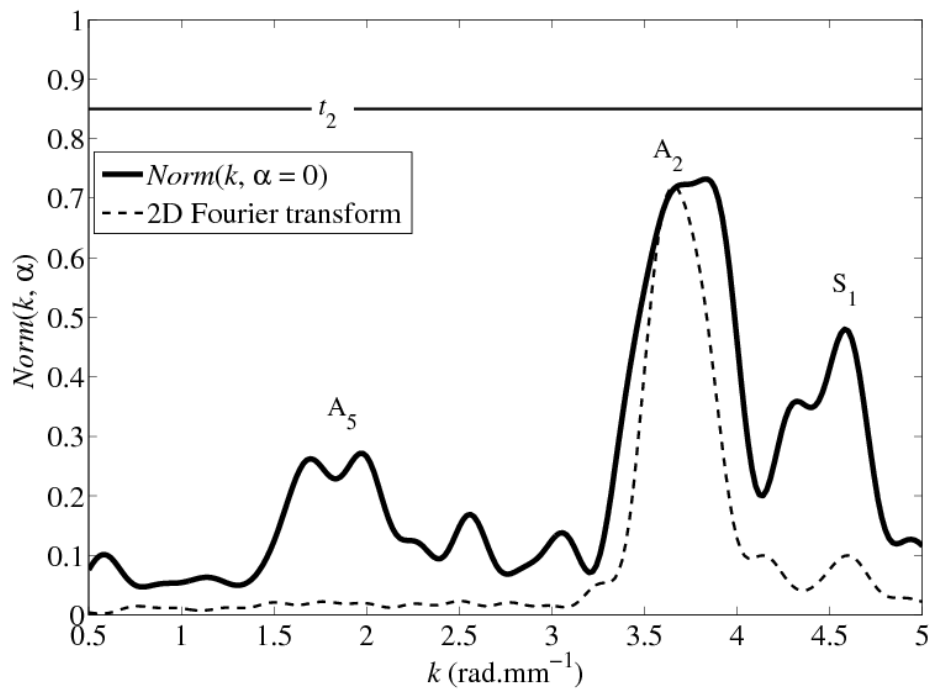

(b)

FIG. 7. $\operatorname{Norm}(f, k, \alpha)$ function in the $(k, \alpha)$ plane for $f=1.84 \mathrm{MHz}(\mathrm{a})$ and for $\alpha=0$ (b), equivalent to the former $\operatorname{Norm}(f, k)$ function formalism. The normalized spatio-temporal Fourier transform is shown in (b) with dashed line. The step between each level line is equal to 0.05 . 
The Norm-function computed under the assumption that the medium is nonattenuating, ${ }^{18}$ i.e., with $\alpha$ equal to 0 , is shown on Fig. 7(b). The same three peaks are visible, but with lower peak values ranging from 0.3 to 0.75 . These values are in agreement with equation (A7). This illustrates how critical are (i) signal-to-noise ratio, and (ii) the choice of the threshold $t_{2}$ to correctly detect the guided modes. In case of noisy data, low value peak may be close to noise peaks and the choice for the second threshold may be difficult. Thus, the extended Norm-function formalism, by taking properly into account the attenuation of the medium, enhances the peak values corresponding to actual guided modes. These peak values can then be easily identified by a proper selection of a high $t_{2}$ threshold value, even for strongly attenuated modes. The spatio-temporal Fourier transform, normalized with the main peak value, is also shown on Fig. 7(b) with dashed line. Only one mode $\left(A_{2}\right)$ is clearly visible. The mode $\mathrm{S}_{1}$ can be confounded with a secondary maximum, and thus is likely not to be detected, depending on its relative value compared to the selected threshold. The most attenuated mode $\mathrm{A}_{5}$ is not measured. Thus the generalized Norm-function appears to be efficient to extract attenuated guided mode wavenumbers using a clinical probe with a limited aperture.

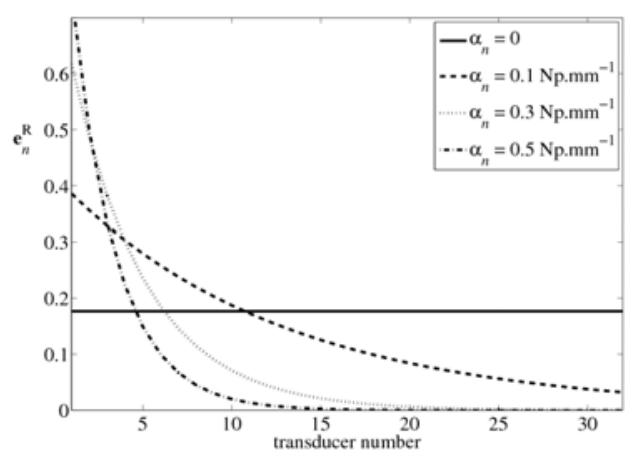

FIG. 8. Modulus of the normalized guided mode $\mathrm{e}_{n}{ }^{\mathrm{R}}\left(k_{n}, \alpha_{n}\right)$ vs. receiver number for three different $\alpha$ values from 0 to 0.5 Np.mm ${ }^{-1}$. 
In this paper, the authors specifically addressed the influence of attenuation on the measurement of guided mode wavenumbers in a bone mimicking plate. The material, is a homogenous absorbing transverse isotropic material which is considered to reasonably match the anisotropic elastic properties of actual human cortical bones. ${ }^{24}$ The disadvantage of performing measurements on phantoms, of course, is that they only approximate true cortical bone structure. In order to generalize the approach to the in vivo measurement conditions, several parameters will have to be taken into account in future studies, such as the irregular geometry, the heterogeneity of the cortical tissue and the presence of marrow and surrounding soft tissues. Performing the measurements with a relatively short reception length of 20-30 mm potentially minimizes the adverse impact of irregular geometry and of bone heterogeneity. The presence of surrounding tissues and marrow will likely affect the wavenumber measurement. However, our preliminary (data not shown) obtained in a bone mimicking plate covered by a soft-tissue mimicking layer suggested that the guided mode wavenumbers are only slightly modified compared to their counterparts in the free bone mimicking plate, and that they are still well predicted by an elastic transverse isotropic waveguide. The authors' intent is to progressively extend the study first to the case of bone phantoms with soft tissue mimicking layers on top of it and marrow inside and then to in vitro experiments with real inhomogeneous bone specimens. These critical issues will be the object of our future studies in order to prepare the in vivo application. 


\section{Conclusion}

An original method for evaluating the complex guided mode wavenumbers, using a probe consisting of two separated emission and reception arrays in contact with the inspected waveguide, is presented in this paper. The method is a generalization of the Norm-function formalism described previously for non attenuated guided mode. This method allows the evaluation of both real and imaginary parts of the attenuated guided mode wavenumbers. Experimental results are in good agreement with the theoretical Lamb modes. The methodology presented herein hold promises for the assessment of the structural and material properties of wave guides, and thus can potentially be used for assessing cortical bone strength. Future studies will focus on the evaluation of elastic properties of cortical bone and will be extended to in vivo measurements. The impact of the geometry (e.g., variable cortical thickness) and soft tissue coupling will also be taken into account.

\section{Acknowledgments}

Authors would like to acknowledge Pierre Nauleau for his help for the transverse transmission measurements and Mohamed Cherrad for the axial transmission measurements.

This work has been supported by ANR project ”COSTUM” 09-TECS-005-03 (2009-2012). 


\section{APPENDIX}

In order to lighten the theoretical part (Sec. II), some notations used are explicitly calculated in this appendix. In particular, the expressions of the norm of the guided mode vector $\mathbf{a}_{n}^{\mathrm{E}}$ and the scalar product $\left\langle\mathbf{e}_{n}{ }^{\mathrm{R}}\right| \mathbf{e}^{\text {test }}>$ are given here. Consider a single attenuated guided mode $\mathbf{a}_{n}{ }^{\mathrm{R}}$ given in Eq. (3). The norm of the $\mathbf{a}_{n}{ }^{\mathrm{R}}$ vector satisfies

$$
\left\|\mathbf{a}_{n}^{\mathrm{R}}\right\|^{2}=\sum_{j=1}^{N^{R}} a_{j n}^{\mathrm{R}} a_{j n}^{\mathrm{R}^{*}}=\sum_{j=1}^{N^{R}} e^{-2 \alpha_{n} \mathrm{x}_{j}^{\mathrm{R}}}
$$

The analytical solution can be evaluated, using an integral solution

$$
\left\|\mathbf{a}_{n}^{\mathrm{R}}\right\|^{2}=\int_{0}^{L} e^{-2 \alpha_{n} x} \frac{d x}{p}
$$

with $p$, the array inter element pitch. Thus, the norm is

$$
\left\|\mathbf{a}_{n}^{\mathrm{R}}\right\|=\sqrt{\frac{N^{\mathrm{R}}}{2 \alpha_{n} L}\left(1-e^{-2 \alpha_{n} L}\right)} .
$$

If $\alpha_{n} L$ tends toward zero, the normalization factor tends toward $\sqrt{N^{\mathrm{R}}}$ as in Ref. ${ }^{18}$. The scalar product between the normalized guided mode $\mathbf{e}_{n}{ }^{\mathrm{R}}$ [Eq. (5)] and the test vector $\mathbf{e}^{\text {test }}$ [Eq. (8)]

$$
\left\langle\mathbf{e}_{n}^{\mathrm{R}}\left(k_{n}, \alpha_{n}\right) \mid \mathbf{e}^{t e s t}(k, \alpha)\right\rangle=\sum_{j=1}^{N^{R}} \frac{1}{\left\|\mathbf{a}^{p w}\right\|\left\|\mathbf{a}_{n}^{\mathrm{R}}\right\|} e^{\left[\mathrm{i}\left(k_{n}-k\right) x_{j}^{\mathrm{R}}-\left(\alpha_{n}+\alpha\right) x_{j}^{\mathrm{R}}\right]} .
$$

In the general case, i.e., $k \neq k_{n}$ and $\alpha \neq \alpha_{n}$, the scalar product is complex. The analytical solution can also be evaluated, using an integral solution

$$
\left\langle\mathbf{e}_{n}^{\mathrm{R}}\left(k_{n}, \alpha_{n}\right) \mid \mathbf{e}^{\text {test }}(k, \alpha)\right\rangle=\frac{1}{\left\|\mathbf{a}^{p w}\right\|\left\|\mathbf{a}_{n}^{\mathrm{R}}\right\|} \int_{0}^{L} e^{(\mathrm{i} \Delta k-\Delta \alpha) x} \frac{d x}{p},
$$

with $\Delta k=k_{n}-k$ and $\Delta \alpha=\alpha_{n}+\alpha$. The solution of the previous equation is

$$
\left\langle\mathbf{e}_{n}^{\mathrm{R}}\left(k_{n}, \alpha_{n}\right) \mid \mathbf{e}^{\text {test }}(k, \alpha)\right\rangle=2 \sqrt{\frac{\alpha_{n} \alpha}{\left(1-e^{-2 \alpha L}\right)\left(1-e^{-2 \alpha_{n} L}\right)}} \frac{1-e^{(\mathrm{i} \Delta k-\Delta \alpha) L}}{\Delta \alpha-\mathrm{i} \Delta k} .
$$


For quasi orthogonal guided modes, the Norm-function is equal to $\left|<\mathbf{e}_{n}{ }^{R}\right| \mathbf{e}^{\text {test }}>\left.\right|^{2}$ [Eq. (13)]. For $k=k_{n}$ and $\alpha=\alpha_{n}$, the scalar product and the Norm-function are maximum and equal to 1 . The Norm-function decrease and lead toward zero as $\Delta k$ and $\Delta \alpha$ increase. With $\alpha_{n}$ and $\alpha$ nulls, the scalar product $<\mathbf{e}_{n}{ }^{\mathrm{R}} \mid \mathbf{e}^{\text {test }}>$ is equal to $e^{\mathrm{i} \Delta k L / 2} \operatorname{sinc}(\Delta k L / 2)$.

The previous Norm-function formalism corresponds to the $\alpha=0$ case and its maximum also corresponds to $k$ equal to $k_{n} \cdot{ }^{18}$ The value of this maximum depends on the $\alpha_{n} L$ product as

$$
\operatorname{Norm}\left(k=k_{n}, \alpha=0\right)=\frac{2\left(1-e^{-\alpha_{n} L}\right)^{2}}{\alpha_{n} L\left(1-e^{-2 \alpha_{n} L}\right)}
$$

Previous equation allows the estimation imaginary part $\alpha_{n}$ from the maximum values of the 2D-Norm(f, $k)$-function. ${ }^{38}$ 
1. P. Moilanen, "Ultrasonic guided waves in bone," IEEE Trans. Ultrason. Ferroelectr. Freq. Control 55, 1277-1286 (2008).

2. M. Talmant, J. Foiret, and J. G. Minonzio, Bone Quantitative ultrasound Chap. 7 Guided waves in cortical bones Springer, New York, pp. 147-179 (2010).

3. A. J. Foldes, A. Rimon, D. D. Keinan, and M. M. Popovtzer, "Quantitative ultrasound of the tibia: A novel approach for assessment of bone status," Bone 17, 363-367 (1995).

4. S. Prevrhal, T. Fuerst, B. Fan, C. Njeh, D. Hans, M. Uffmann, S. Srivastav, and H. K. Genant, "Quantitative ultrasound of the tibia depends on both cortical density and thickness," Osteoporos. Int. 12, 28-34 (2001).

5. H. Sievanen, S. Cheng, S. Ollikainen, and K. Uusi-Rasi, "Ultrasound velocity and cortical bone characteristics in vivo," Osteoporos. Int. 12, 399-405 (2001).

6. V. Kilappa, P. Moilanen, L. Xu, P. H. F. Nicholson, J. Timonen, and S. Cheng, "Lowfrequency axial ultrasound velocity correlates with bone mineral density and cortical thickness in the radius and tibia in pre- and postmenopausal women," Osteoporos. Int. 22, 1103-1113 (2011).

7. D. Hans, S. K. Srivastav, C. Singal, R. Barkmann, C. F. Njeh, E. Kantorovich, C. C. Gluer, and H. K. Genant, "Does combining the results from multiple bone sites measured by a new quantitative ultrasound device improve discrimination of hip fracture?," J. Bone Miner. Res. 14, 644-651 (1999).

8. M. Talmant, S. Kolta, C. Roux, D. Haguenauer, I. Vedel, B. Cassou, E. Bossy, and P. Laugier, "In Vivo Performance Evaluation of Bi-Directional Ultrasonic Axial Transmission for Cortical Bone Assessment," Ultrasound Med. Biol. 35, 912-919 (2009). 
9. A. Sarvazyan, A. Tatarinov, V. Egorov, S. Airapetian, V. Kurtenok, and C. J. Gatt, "Application of the dual-frequency ultrasonometer for osteoporosis detection," Ultrasonics 49, 331-337 (2009).

10. P. Moilanen, P. H. F. Nicholson, T. Karkkainen, Q. Wang, J. Timonen, and S. Cheng, "Assessment of the tibia using ultrasonic guided waves in pubertal girls," Osteoporos. Int. 14, 1020-1027 (2003).

11. P. Moilanen, M. Talmant, V. Kilappa, P. Nicholson, S. L. Cheng, J. Timonen, and P. Laugier, "Modeling the impact of soft tissue on axial transmission measurements of ultrasonic guided waves in human radius," J. Acoust. Soc. Am. 124, 2364-2373 (2008).

12. F. Lefebvre, Y. Deblock, P. Campistron, D. Ahite, and J. J. Fabre, "Development of a new ultrasonic technique for bone and biomaterials in vitro characterization," J. Biomed. Mater. Res. 63, 441-446 (2002).

13. D. Ta, W. Q. Wang, Y. Y. Wang, L. H. Le, and Y. Q. Zhou, "Measurement of the Dispersion and Attenuation of Cylindrical Ultrasonic Guided Waves in Long Bone," Ultrasound Med. Biol. 35, 641-652 (2009).

14. L. H. Le, Y. J. Gu, Y. P. Li, and C. Zhang, "Probing long bones with ultrasonic body waves," Appl. Phys. Lett. 96, 114102 (2010).

15. V. C. Protopappas, D. I. Fotiadis, and K. N. Malizos, "Guided ultrasound wave propagation in intact and healing long bones," Ultrasound Med. Biol. 32, 693-708 (2006).

16. D. A. Ta, K. Huang, W. Q. Wang, Y. Y. Wang, and L. H. Le, "Identification and analysis of multimode guided waves in tibia cortical bone," Ultrasonics 44, E279E284 (2006). 
17. K. L. Xu, D. A. Ta, and W. Q. Wang, "Multiridge-Based Analysis for Separating Individual Modes From Multimodal Guided Wave Signals in Long Bones," IEEE Trans. Ultrason. Ferroelectr. Freq. Control 57, 2480-2490 (2010).

18. J. G. Minonzio, M. Talmant, and P. Laugier, "Guided wave phase velocity measurement using multi-emitter and multi-receiver arrays in the axial transmission configuration," J. Acoust. Soc. Am. 127, 2913-2919 (2010).

19. J. G. Minonzio, M. Talmant, and P. Laugier, "Measurement of guided mode wave vectors by analysis of the transfer matrix obtained with multi-emitters and multireceivers in contact," Journal of Physics: Conference Series 269, 012003 (2011).

20. E. Bossy, M. Talmant, M. Defontaine, F. Patat, and P. Laugier, "Bidirectional axial transmission can improve accuracy and precision of ultrasonic velocity measurement in cortical bone: A validation on test materials," IEEE Trans. Ultrason. Ferroelectr. Freq. Control 51, 71-79 (2004).

21. I. A. Viktorov, Rayleigh and Lamb waves Plenum Press, New York, NY, pp.82 -89 (1967).

22. E. Moulin, S. Grondel, M. Baouahi, and J. Assaad, "Pseudo-3D modeling of a surfacebonded Lamb wave source (L)," J. Acoust. Soc. Am. 119, 2575-2578 (2006).

23. Y. Hua and T. K. Sarkar, "Matrix Pencil Method for Estimating Parameters of Exponentially Damped Undamped Sinusoids in Noise," IEEE Trans. Acoust. Speech, Signal Processing 38, 814-824 (1990).

24. M. Granke, Q. Grimal, A. Saïed, P. Nauleau, F. Peyrin, and P. Laugier, "Change in porosity is the major determinant of the variation of cortical bone elasticity at the millimeter scale in aged women," Bone In Press ( (2011).

25. W. Sachse and Y. H. Pao, "Determination of Phase and Group Velocities of Dispersive Waves in Solids," Journal of Applied Physics 49, 4320-4327 (1978). 
26. S. Chaffai, F. Peyrin, S. Nuzzo, R. Porcher, G. Berger, and P. Laugier, "Ultrasonic characterization of human cancellous bone using transmission and backscatter measurements: Relationships to density and microstructure," Bone 30, 229-237 (2002).

27. F. Peters and L. Petit, "A broad band spectroscopy method for ultrasound wave velocity and attenuation measurement in dispersive media," Ultrasonics 41, 357-363 (2003).

28. T. L. Szabo, "Causal theories and data for acoustic attenuation obeying a frequency power law," J. Acoust. Soc. Am. 97, 14-24 (1995).

29. M. Treiber, J. Y. Kim, L. J. Jacobs, and J. M. Qu, "Correction for partial reflection in ultrasonic attenuation measurements using contact transducers," J. Acoust. Soc. Am. 125, 2946-2953 (2009).

30. B. Hartmann and J. Jarzynsk, "Ultrasonic Hysteresis Absorption in Polymers," J. Appl. Phys. 43, 4304-4312 (1972).

31. B. Hosten, L. Moreau, and M. Castaings, "Reflection and transmission coefficients for guided waves reflected by defects in viscoelastic material plates," J. Acoust. Soc. Am. 121, 3409-3417 (2007).

32. R. Longo, Q. Grimal, P. Laugier, S. Vanlanduit, and P. Guillaume, "Simultaneous Determination of Acoustic Velocity and Density of a Cortical Bone Slab: Ultrasonic Model-Based Approach," IEEE Trans. Ultrason. Ferroelectr. Freq. Control 57, 496500 (2010).

33. S. P. Dodd, J. L. Cunningham, A. W. Miles, S. Gheduzzi, and V. F. Humphrey, "Ultrasonic propagation in cortical bone mimics," Phys. Med. and Biol. 51, 46354647 (2006). 
34. Y. J. Wang, R. E. Challis, A. P. Y. Phang, and M. E. Unwin, "Bulk Shear Wave Propagation in an Epoxy: Attenuation and Phase Velocity over Five Decades of Frequency," IEEE Trans. Ultrason. Ferroelectr. Freq. Control 56, 2504-2513 (2009).

35. G. A. Coquin, "Attenuation of Guided Waves in Isotropic Viscoelastic Materials," J.Acoust. Soc. Am. 36, 1074-1080 (1964).

36. C. W. Chan and P. Cawley, "Lamb waves in highly attenuative plastic plates," J. Acoust. Soc. Am. 104, 874-881 (1998).

37. S. H. Rhee, J. K. Lee, and J. J. Lee, "The group velocity variation of Lamb wave in fiber reinforced composite plate," Ultrasonics 47, 55-63 (2007).

38. J. G. Minonzio, J. Foiret, M. Talmant, and P. Laugier, "Guided wave phase velocity measurement in anisotropic absorbing material " IEEE Ultrasonics Symposium (IUS), pp. 2159-2162 (2010). 
TABLE I. Measured values for PMMA and bone mimicking (bone mim.) plates

\begin{tabular}{lcccccc}
\hline & $c_{\mathrm{L}}\left(\mathrm{m} . \mathrm{s}^{-1}\right)$ & $c_{\mathrm{T}}\left(\mathrm{m} . \mathrm{s}^{-1}\right)$ & $(\alpha \lambda)_{\mathrm{L}}(\mathrm{Np})$ & $(\alpha \lambda)_{\mathrm{T}}(\mathrm{Np})$ & $\rho\left(\mathrm{g} . \mathrm{cm}^{-3}\right)$ & $e(\mathrm{~mm})$ \\
\hline \hline PMMA & $2685 \pm 20$ & $1365 \pm 10$ & $0.059 \pm 0.007$ & $0.063 \pm 0.003$ & 1.18 & 1.95 \\
bone mim. $\perp$ & $2870 \pm 40$ & $1520 \pm 20$ & $0.087 \pm 0.013$ & $0.147 \pm 0.004$ & 1.64 & 2.30 \\
bone mim. $\|$ & $3705 \pm 40$ & $1585 \pm 20$ & & & & \\
& & & & & & \\
\hline
\end{tabular}


FIG. 1. Geometry of the problem: emission and reception arrays are placed in the axial transmission configuration.

FIG. 2. Schematic representation of a signal subspace of rank 2: the test vector $\mathbf{e}^{\text {test }}$ can be projected on the singular vector basis $\left(\mathbf{U}_{1}, \mathbf{U}_{2}\right)$ [Eq. (9)] or in normalized guided mode basis $\left(\mathbf{e}_{1}{ }^{\mathrm{R}}, \mathbf{e}_{2}{ }^{\mathrm{R}}\right)$ [Eq. (11)].

FIG. 3. Results for the PMMA plate: real (a) and imaginary parts (b) of the guided mode wavenumbers $k_{n}(f)+\mathrm{i} \alpha_{n}(f)$. Experimental values (each mode is represented with a different symbol) determined from the 3D Norm values and a threshold $t_{2}=0.85$ are compared with the theoretical Lamb modes $\mathrm{A}_{n}$ (continuous line) and $\mathrm{S}_{n}$ (dash line). For the imaginary part, four modes are shown $S_{1}, S_{2}, S_{4}$ and $A_{5}$. Arrows correspond to guided mode excitability minima. The $f=1.84 \mathrm{MHz}$ case (shown with a vertical dash-dot line) is discussed in paragraph IV.C.

FIG. 4. Intrinsic excitability $E_{n}$ function [Eq. (2)] of a $1.95 \mathrm{~mm}$ thick PMMA plate for the $\mathrm{S}_{1}$ and $\mathrm{S}_{2}$ modes. If the excitability is too low (as shown by the arrows), the modes are not observed.

FIG. 5. Results for the $2.3 \mathrm{~mm}$ bone mimicking plate, measured with the probe oriented perpendicularly to the fibers. Real (a) and imaginary (b) parts of the guided mode wavenumbers $k_{n}(f)+\mathrm{i} \alpha_{n}(f)$.

FIG. 6. Results for the $2.3 \mathrm{~mm}$ bone mimicking plate, the probe parallel to the fibers, real part $k_{n}(f)$ of the guided mode wavenumbers. 
FIG. 7. $\operatorname{Norm}(f, k, \alpha)$ function in the $(k, \alpha)$ plane for $f=1.84 \mathrm{MHz}$ (a) and for $\alpha=0$ (b), equivalent to the former $\operatorname{Norm}(f, k)$ function formalism. The normalized spatio-temporal Fourier transform is shown in (b) with dashed line. The step between each level line is equal to 0.05 .

FIG. 8. Modulus of the normalized guided mode $\mathrm{e}_{n}{ }^{\mathrm{R}}\left(k_{n}, \alpha_{n}\right)$ vs. receiver number for three different $\alpha$ values from 0 to 0.5 Np.mm ${ }^{-1}$. 


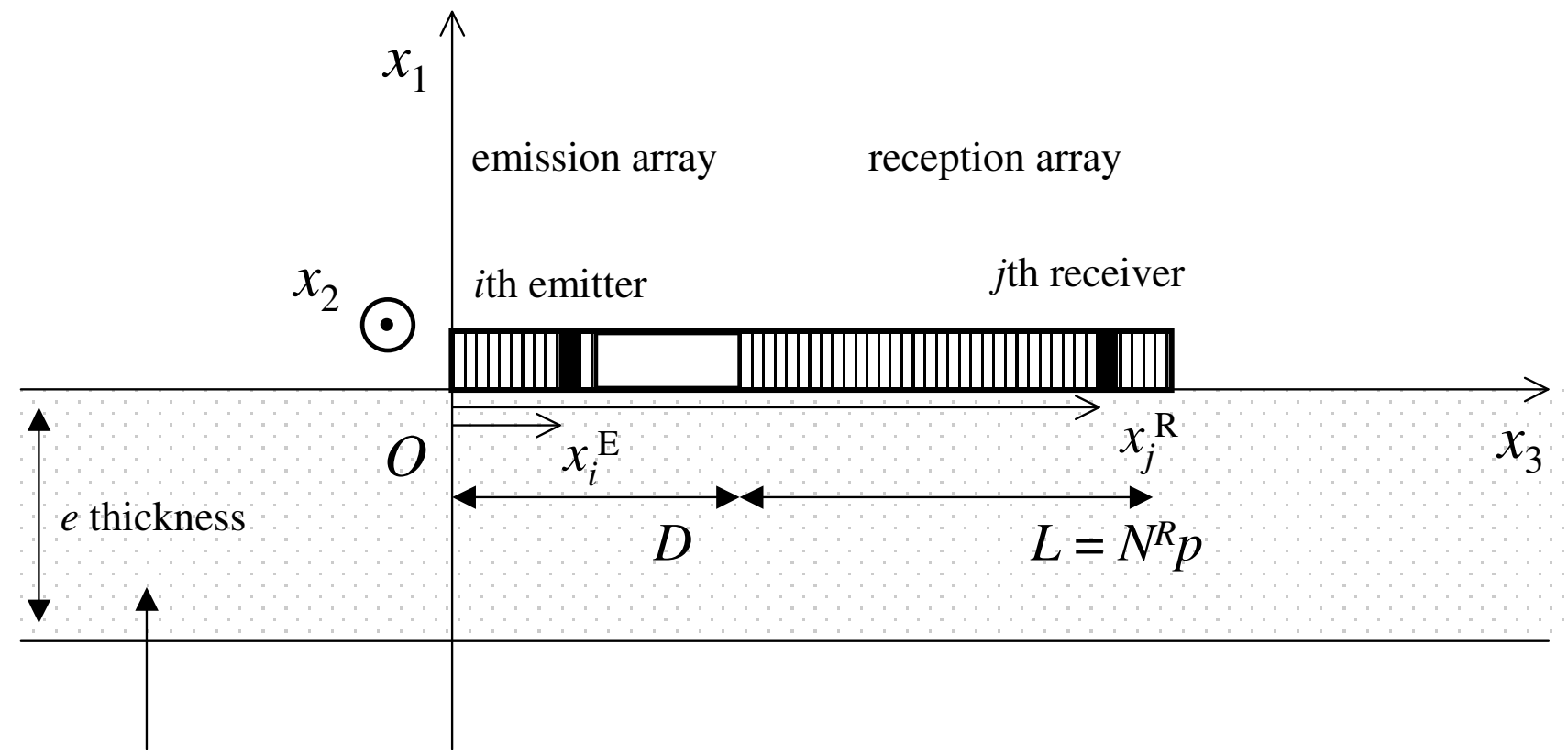

propagating medium :

may be absorbing and/or anistropic 


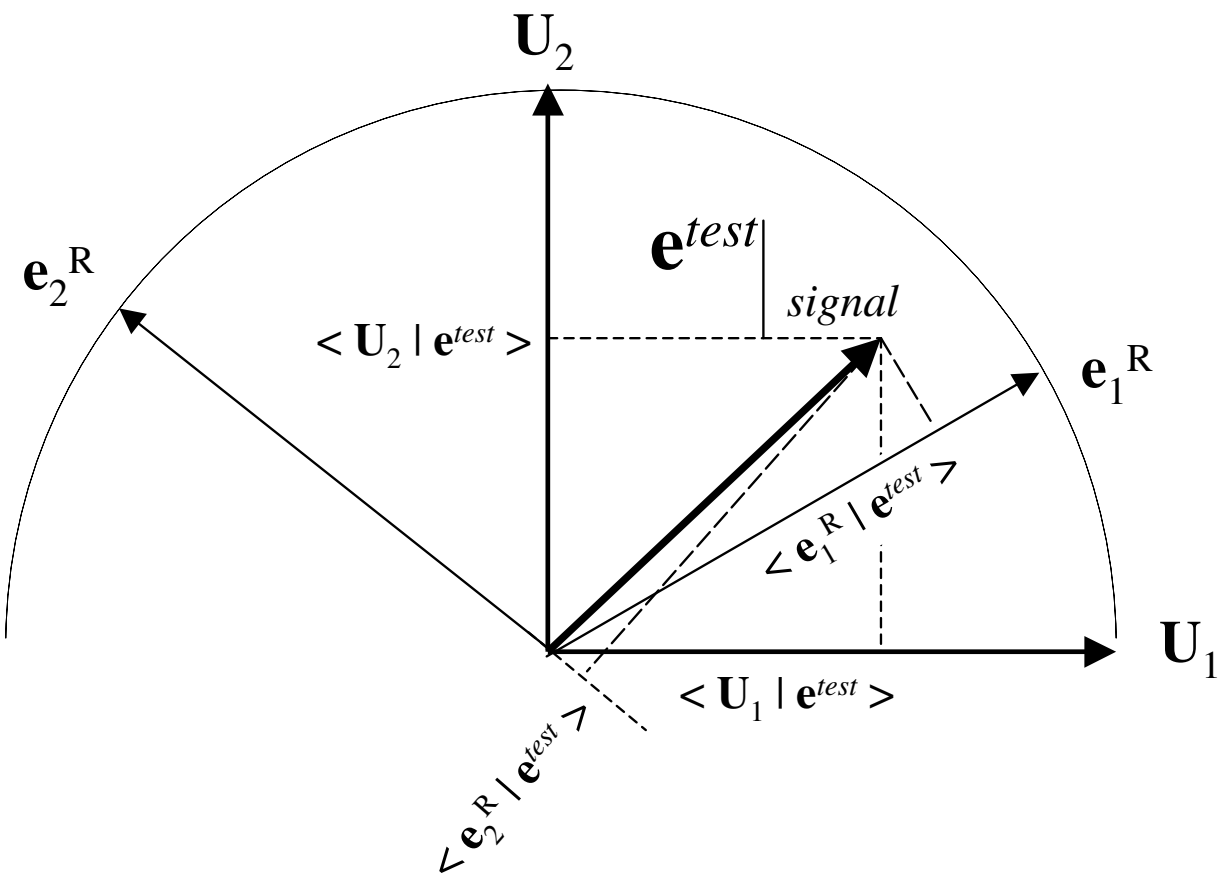




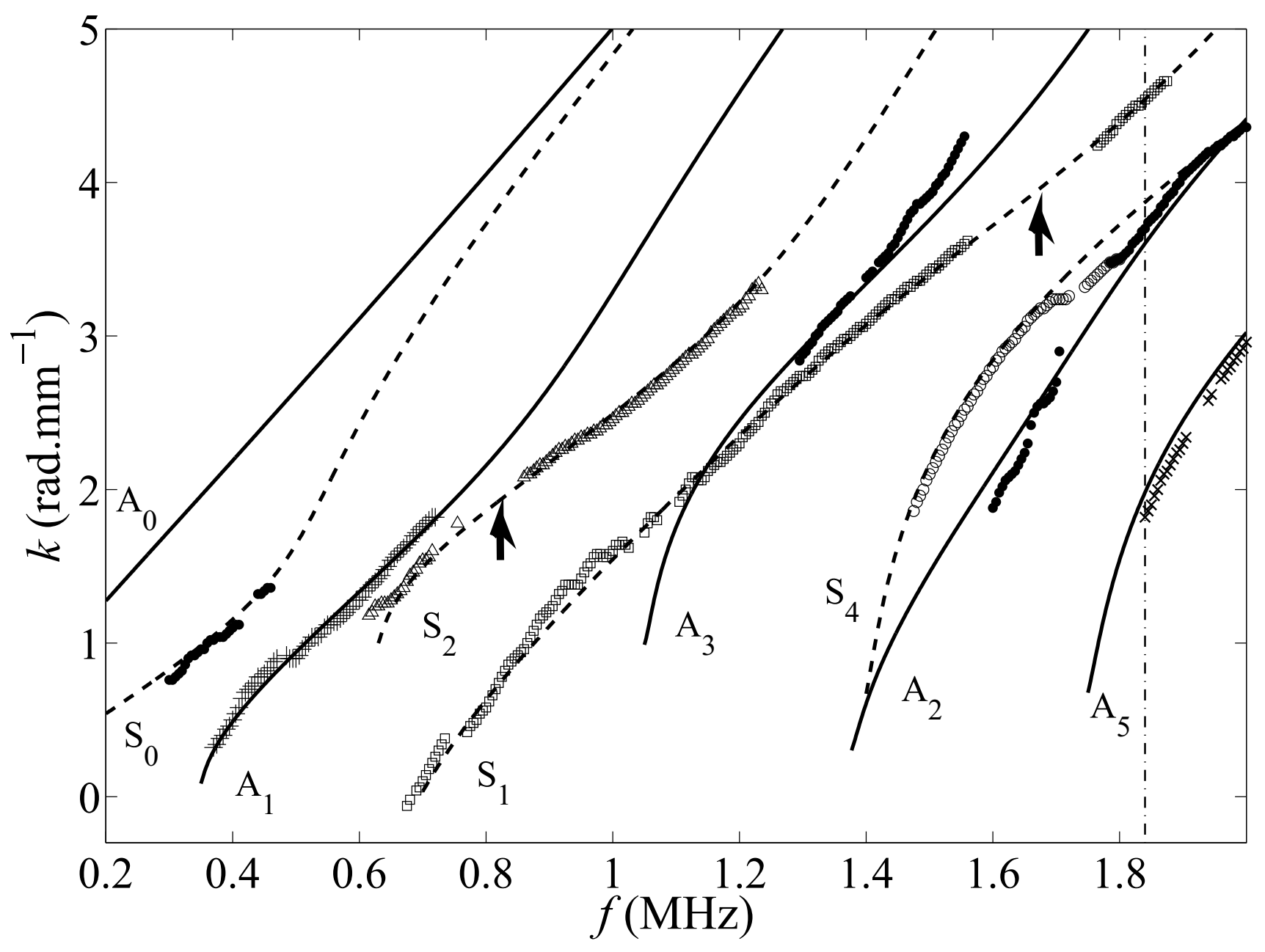




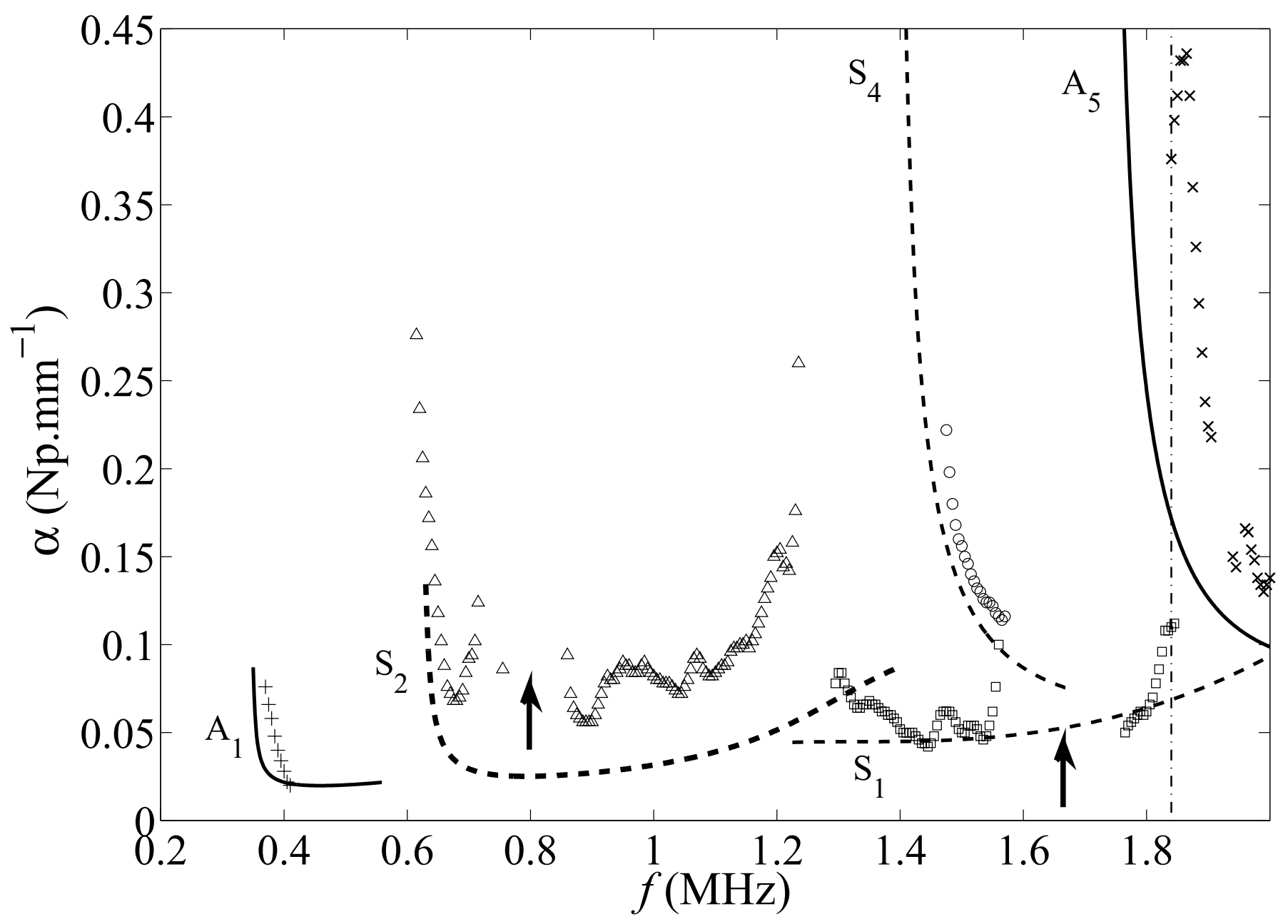




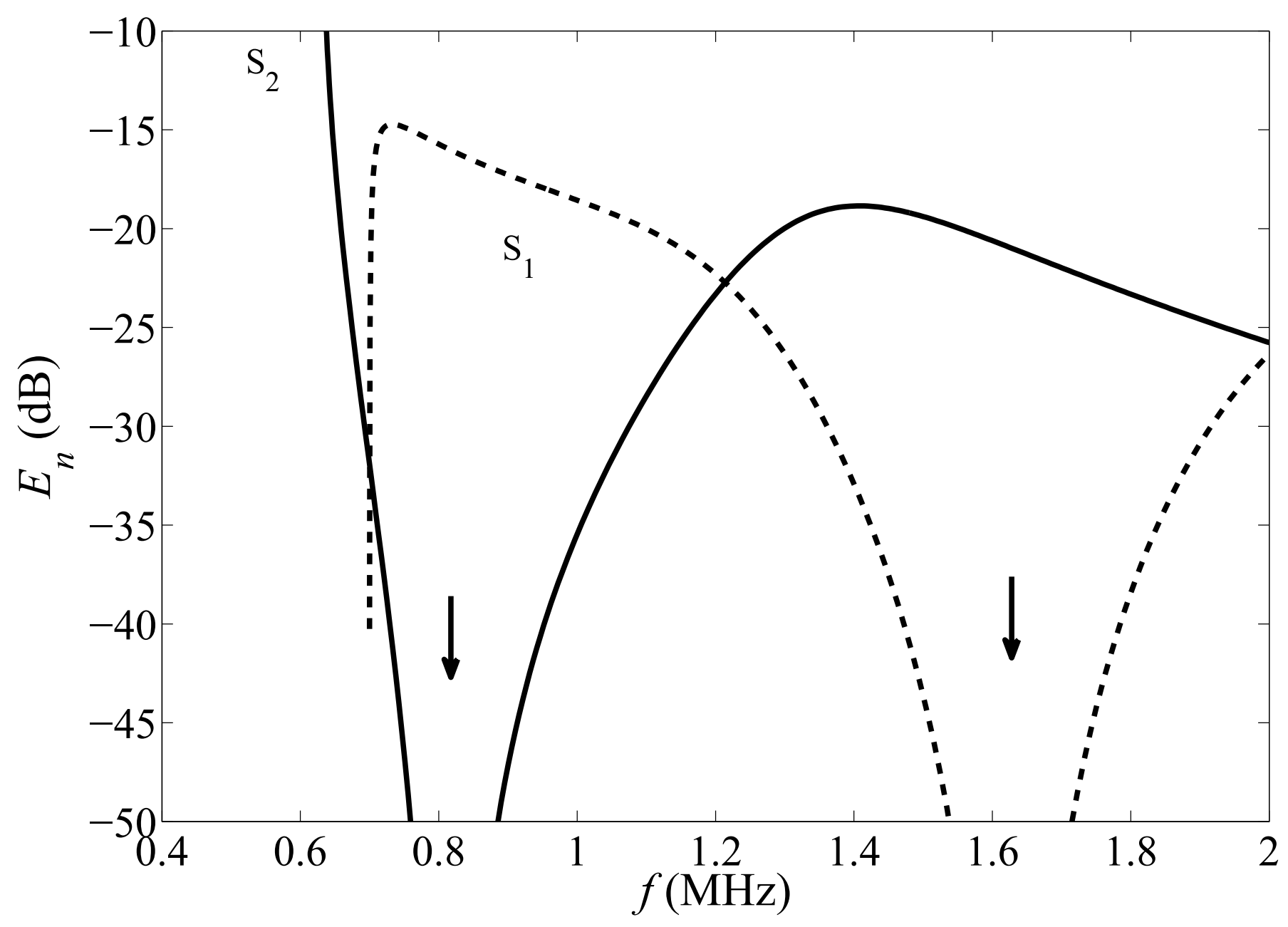




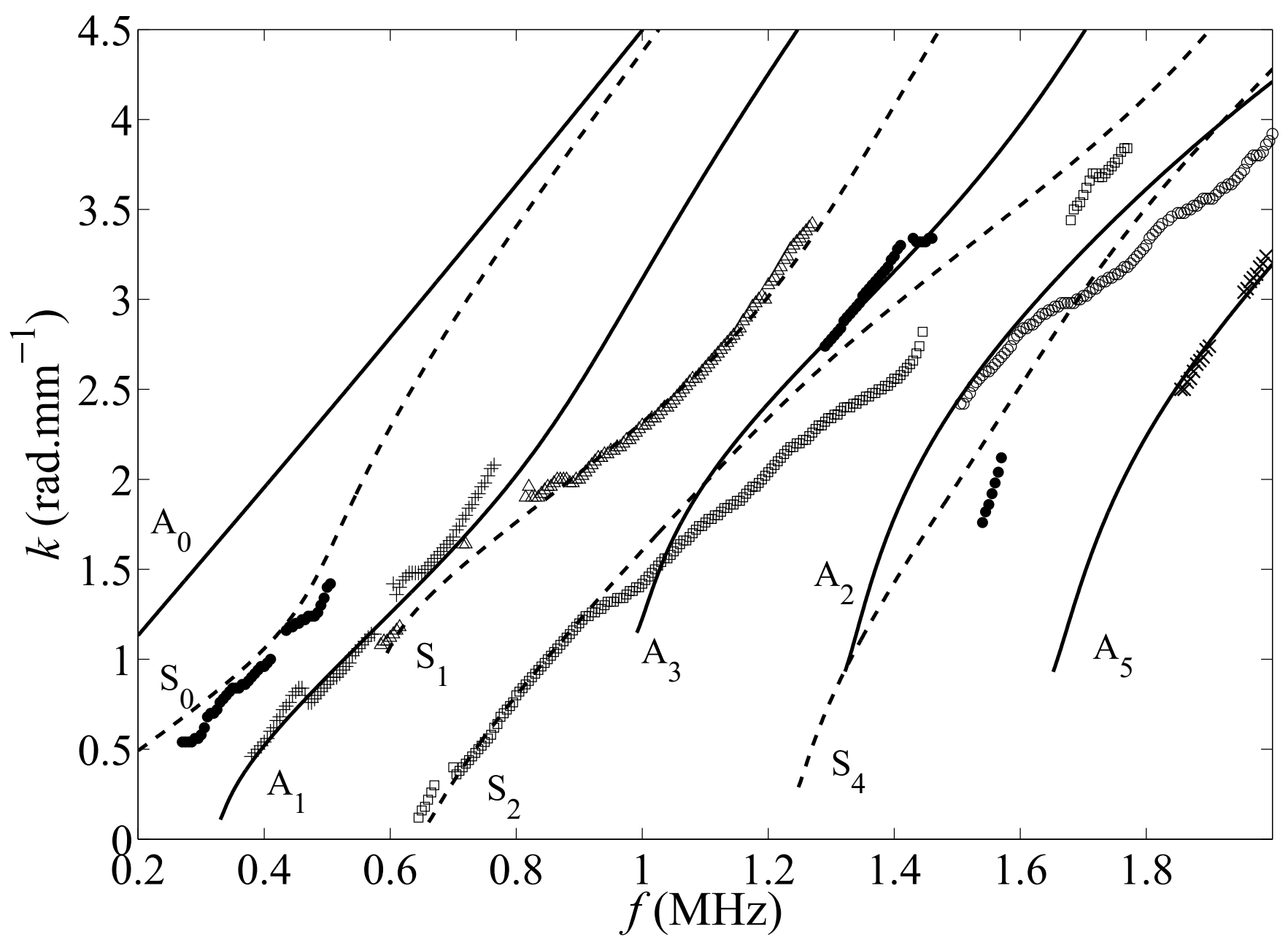




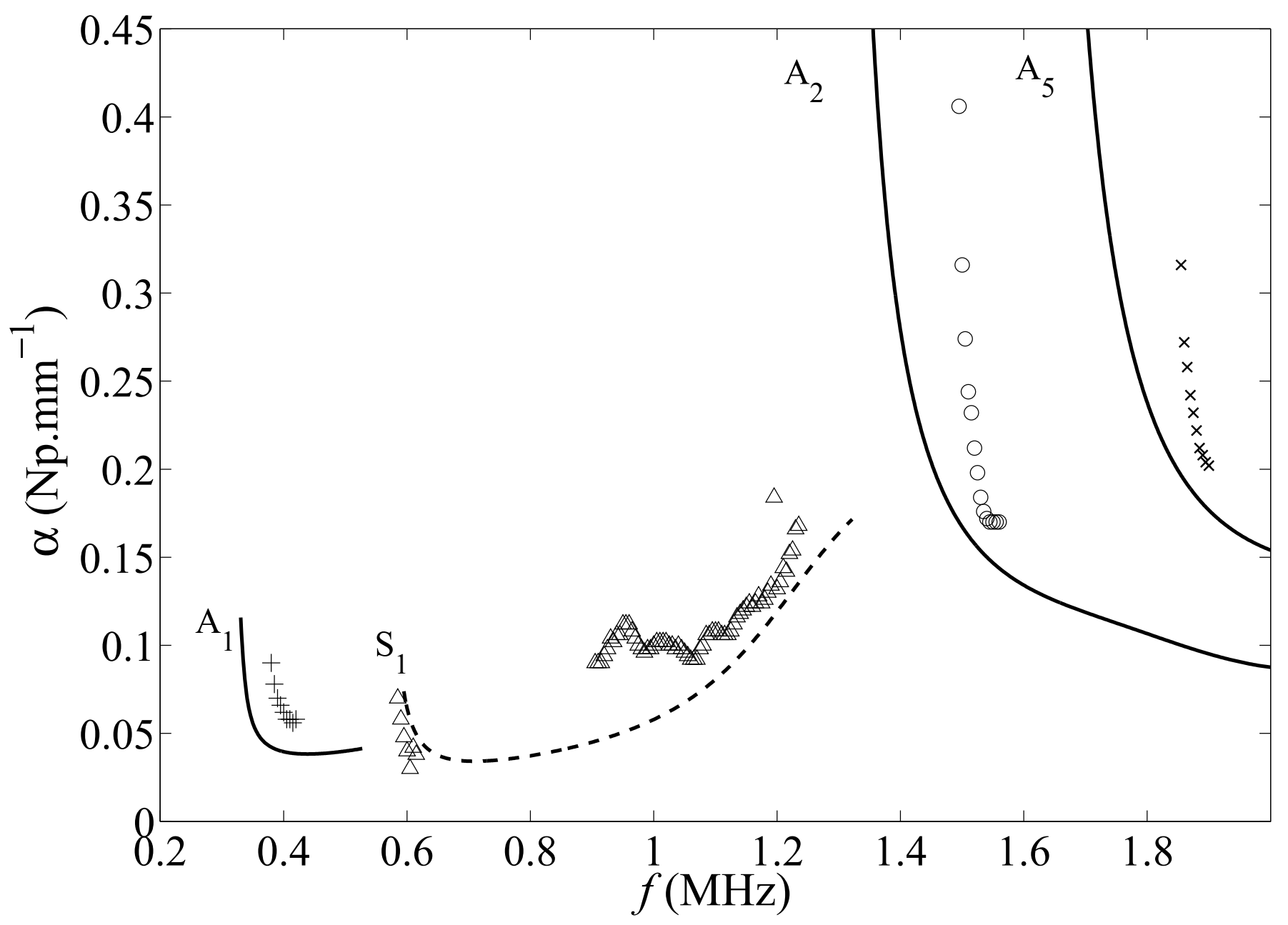




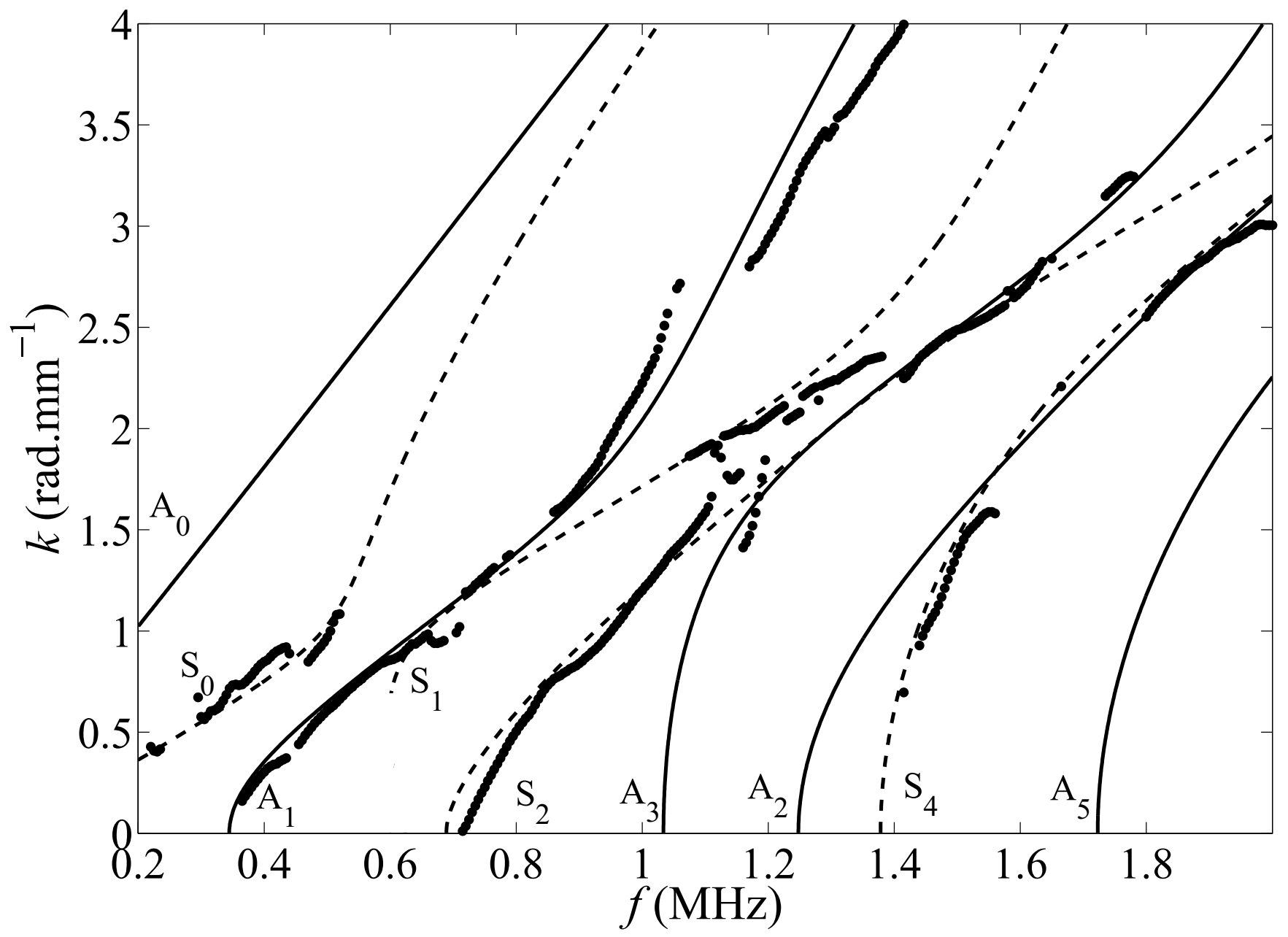




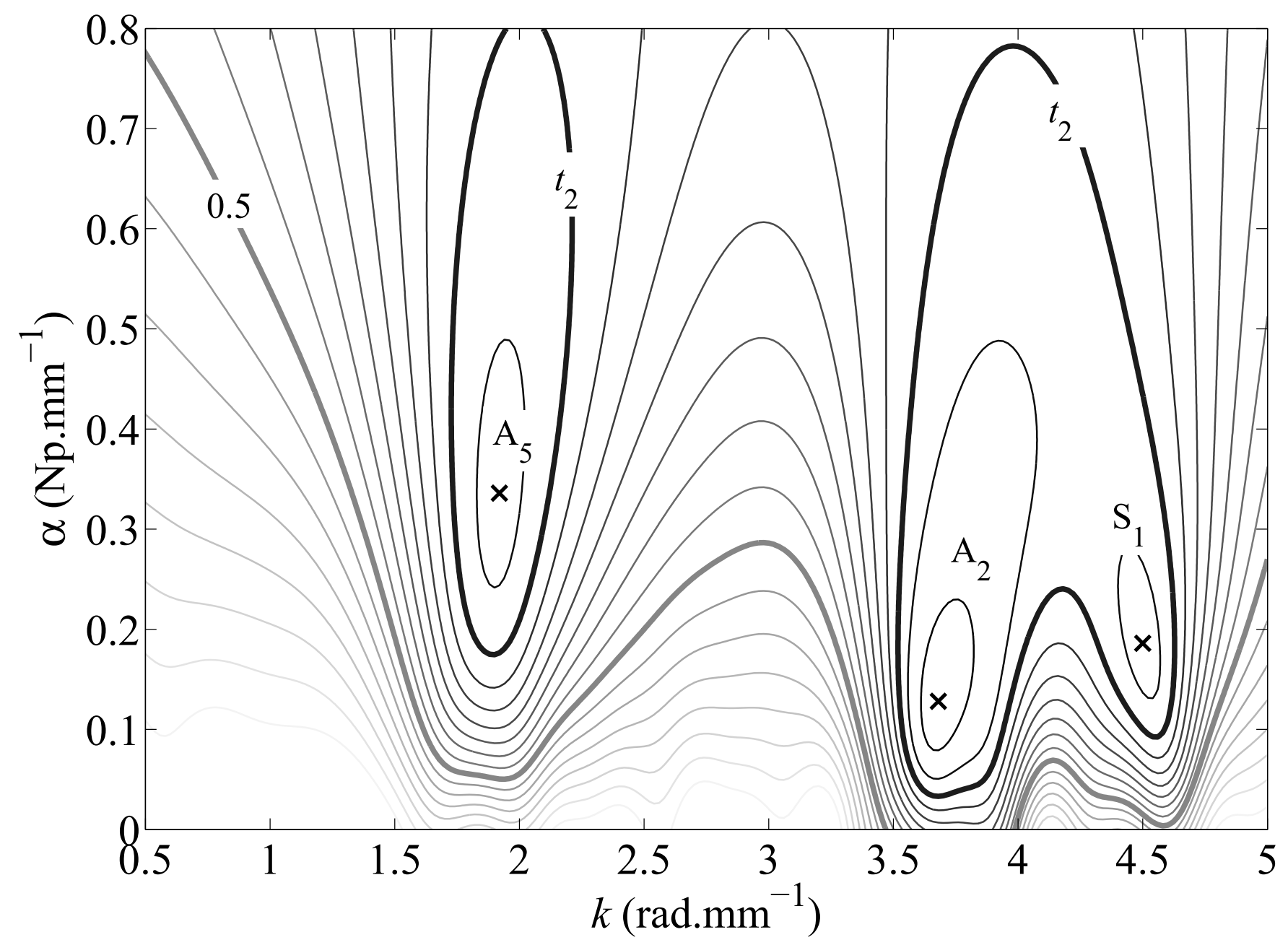




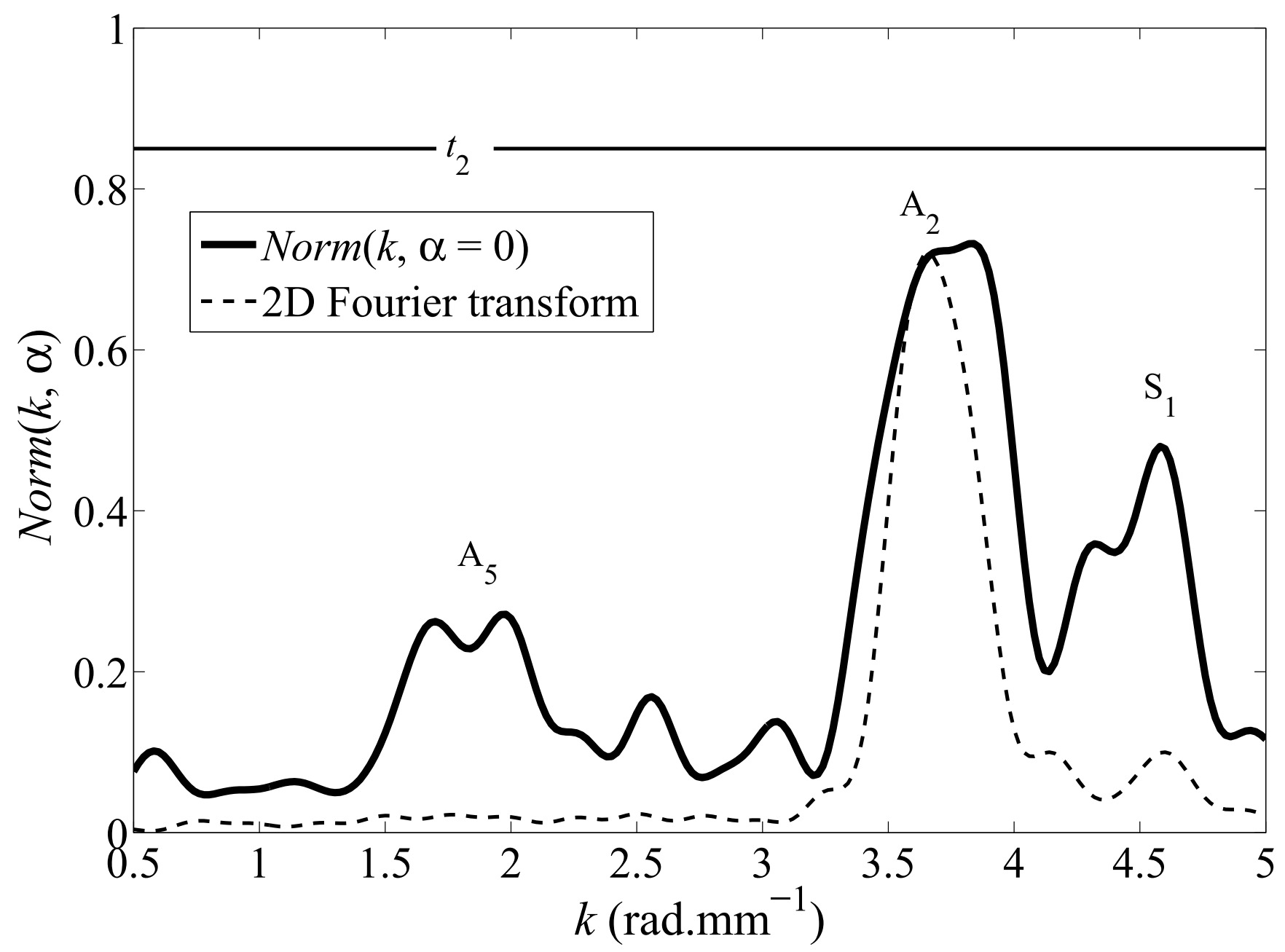




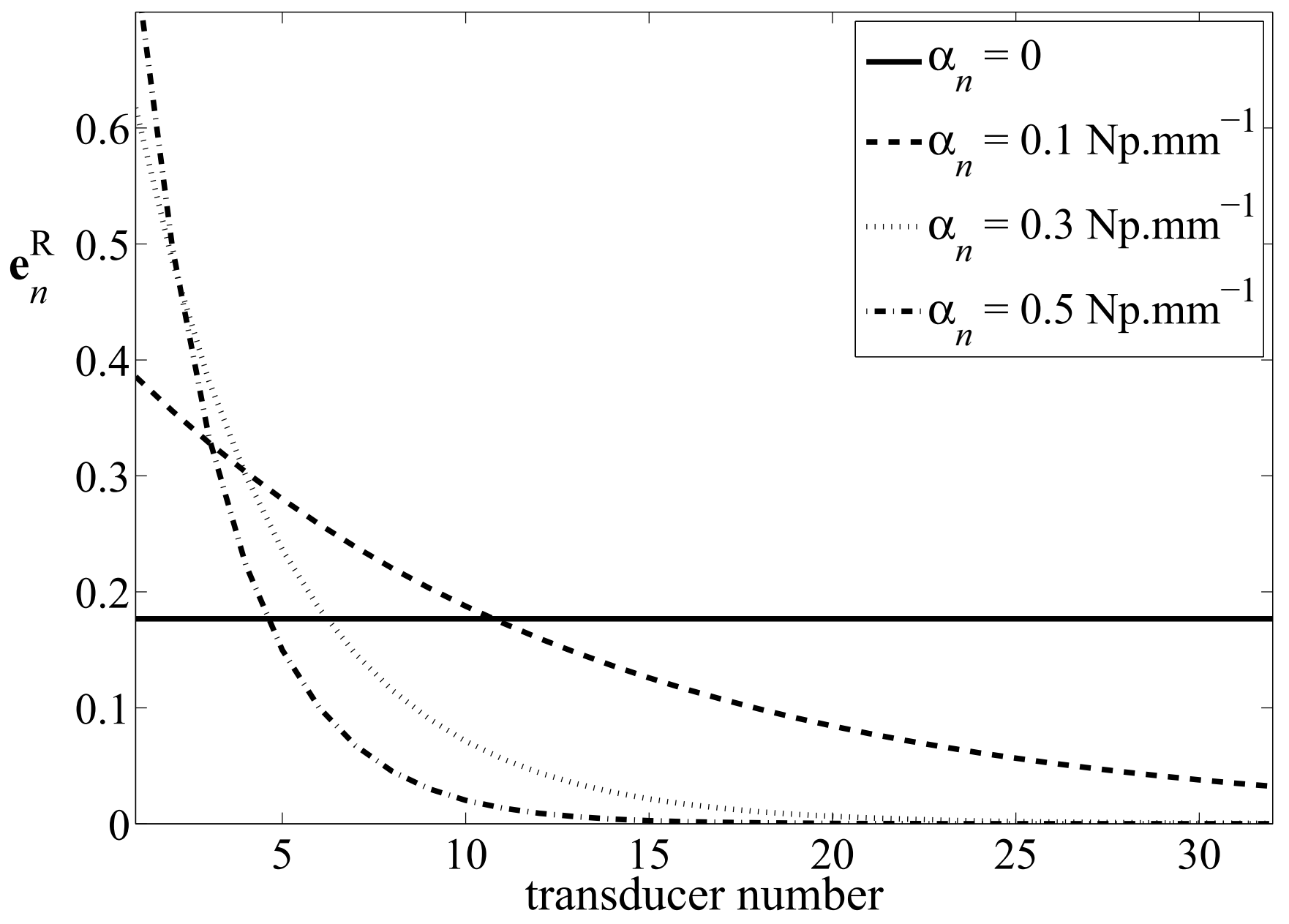

